Proc. IFAC Conf. Nonlinear Control (NOLCOS), Bologna, 2010, p.1-29.

\title{
Discontinuous Feedback and Nonlinear Systems *
}

\author{
Francis Clarke* \\ * Université de Lyon, Institut Camille Jordan \\ 69622 Villeurbanne, France (e-mail: clarke@math.univ-lyon1.fr)
}

\begin{abstract}
This tutorial paper is devoted to the controllability and stability of control systems that are nonlinear, and for which, for whatever reason, linearization fails. We begin by motivating the need for two seemingly exotic tools: nonsmooth control-Lyapunov functions, and discontinuous feedbacks. Then, after a (very) short course on nonsmooth analysis, we build a theory around these tools. We proceed to apply it in various contexts, focusing principally on the design of discontinuous stabilizing feedbacks.
\end{abstract}

Keywords: controllability, discontinuous control, feedback, nonlinear theory, stabilization

\section{INTRODUCTION}

Our interest centers throughout on the standard control system

$$
x^{\prime}(t)=f(x(t), u(t)) \text { a.e., } u(t) \in U \text { a.e., }
$$

where the dynamics function $f: \mathbb{R}^{n} \times \mathbb{R}^{m} \rightarrow \mathbb{R}^{n}$ and the control set $U \subset \mathbb{R}^{m}$ are given, and 'a.e.' is the abbreviation of 'almost everywhere'. A control on some interval $[a, b]$ of interest refers to a measurable function $u(\cdot)$ defined on $[a, b]$ and having values in $U$. By a trajectory of the system $(*)$ we mean (as usual) an absolutely continuous state function $x:[a, b] \rightarrow \mathbb{R}^{n}$ corresponding to some choice of control $u(\cdot)$.

Standing hypotheses. It is assumed throughout that $f$ is continuous, $U$ is compact, and $f$ is locally Lipschitz with respect to the state variable in the following sense: for every bounded subset $S \subset \mathbb{R}^{n}$, there exists $K=K_{f}(S)$ such that

$$
|f(x, u)-f(y, u)| \leq K_{f}|x-y| \quad \forall x, y \in S, u \in U .
$$

We remark that this Lipschitz behavior is automatically present if $f$ is continuously differentiable, but differentiability of $f$ is irrelevant to our discussion. Much more to the point are the assumptions that are not being made: $f$ is not linear, $U$ is not simply 'large enough' to be effectively ignored.

The central issue under discussion will be the convergence of state trajectories $x(t)$ to an equilibrium, which we take to be the origin: stability, controllability, and feedback stabilization. Stabilization to the origin is a simple representative of various other objectives that can be treated by the techniques that we shall describe. (Stabilization to other target sets will also be involved later.)

One way to steer trajectories to zero is to invent a cost whose minimization will have that effect. (Indeed, in a certain sense, this is rather close to being the only effective

* Chaire en théorie mathématique du contrôle Institut universitaire de France. strategy that we know.) The positive features of such an approach, as well as certain inherent difficulties which arise when we employ it, are well illustrated by what is called the dynamic programming technique in optimal control. It will furnish us with valuable insight into our stabilization problem, and provide guidance about the mathematical tools needed.

\section{Dynamic programming and minimal time}

The minimal-time problem refers to finding a trajectory of $(*)$ that reaches the origin as quickly as possible from a given initial point $\alpha$. Thus we seek the least $T \geq 0$ admitting a control function $u(\cdot)$ on $[0, T]$ having the property that the resulting trajectory $x$ with $x(0)=\alpha$ satisfies $x(T)=0$. The dynamic programming approach centers upon the minimal-time function $T(\cdot)$, defined on $\mathbb{R}^{n}$ as follows: $T(\alpha)$ is the least time $T$ defined above.

The principle of optimality makes two observations about $T(\cdot)$. The first of these is that, for any trajectory $x(\cdot)$ beginning at $\alpha$, for any two times $s, t$ with $0 \leq s<t$, we have

$$
T(x(s)) \leq T(x(t))+t-s .
$$

This reflects the fact that, starting at the point $x(s)$, we may choose the two-step strategy of following the trajectory $x$ until time $t$, and then proceeding optimally from the point $x(t)$ to the origin. The time required for this two-step strategy is the right side of (2); the inequality holds because there may be a better strategy beginning from $x(s)$.

The second observation is that equality holds in (2) if $x$ is a trajectory that joins $\alpha$ to the origin in minimal time; that is, if $x(T)=0$ for $T=T(\alpha)$. This reflects the fact that when $x$ is a minimal-time trajectory, there is no better strategy than the two-step one described above.

Combining these two observations, we find that, for any trajectory $x(\cdot)$, the function $t \mapsto T(x(t))+t$ is nondecreasing; it is constant when $x$ is a minimal-time trajectory. 
Since $t \mapsto T(x(t))+t$ is nondecreasing, we expect to have

$$
\left\langle\nabla T(x(t)), x^{\prime}(t)\right\rangle+1 \geq 0,
$$

with equality when $x(\cdot)$ is an optimal trajectory. The possible values of $x^{\prime}(t)$ for a trajectory being precisely the elements of the set $f(x(t), U)$, we arrive at

$$
\min _{u \in U}\langle\nabla T(x), f(x, u)\rangle+1=0 .
$$

We define the (lower) Hamiltonian function $h$ as follows:

$$
h(x, p):=\min _{u \in U}\langle p, f(x, u)\rangle .
$$

In terms of $h$, the partial differential equation (3) above reads

$$
h(x, \nabla T(x))+1=0,
$$

a special case of the Hamilton-Jacobi equation.

We have now reached the first stage in the dynamic programming approach: solve the Hamilton-Jacobi equation (5), together with the boundary condition $T(0)=0$, to find $T(\cdot)$. How will this help us find minimal-time trajectories?

To answer this question, we recall that an optimal trajectory is such that equality holds in (3). This suggests the following procedure: For each $x$, let $k(x)$ be a point in $U$ satisfying

$$
\min _{u \in U}\langle\nabla T(x), f(x, u)\rangle=\langle\nabla T(x), f(x, k(x))\rangle=-1 .
$$

Then, if we construct $x(\cdot)$ via the initial-value problem

$$
x^{\prime}(t)=f(x(t), k(x(t))), \quad x(0)=\alpha,
$$

we obtain a minimum-time trajectory (from $\alpha$ ).

Let us see why this so: If $x(\cdot)$ satisfies (7), then, in light of (6), we have

$$
\begin{aligned}
(d / d t) T(x(t)) & =\left\langle\nabla T(x(t)), x^{\prime}(t)\right\rangle \\
& =\langle\nabla T(x(t)), f(x(t), k(x(t)))\rangle=-1 .
\end{aligned}
$$

Integrating, we find

$$
T(x(t))=T(\alpha)-t,
$$

which implies that at $\tau=T(\alpha)$, we have $T(x(\tau))=0$, whence $x(\tau)=0$ (since $T$ is zero only at the origin). Therefore $x(\cdot)$ is a minimal-time trajectory.

This second stage of the dynamic programming approach has provided a feedback $k(\cdot)$ which, from any initial value $\alpha$, generates via (7) a minimal-time trajectory; $k$ constitutes what can be considered the ultimate solution to our problem: an optimal feedback synthesis.

We remark that the Hamilton-Jacobi equation (5) has another use, when we know that it has a unique solution $T(\cdot)$ satisfying $T(0)=0$ (namely, the minimal-time function). We refer to the verification method in optimal control (see for example Clarke (1989)). It would work here as follows: Suppose we have formulated a conjecture that, for each $\alpha$, a certain trajectory $x_{\alpha}$ is a minimal-time one from the initial condition $\alpha$. We proceed to calculate $T(\alpha)$ (provisionally) based on this conjecture; that is, by setting $T(\alpha)$ equal to the time required for $x_{\alpha}$ to join $\alpha$ to 0 . Then, if the resulting function $T$ satisfies (5), our conjecture is verified (since, by uniqueness, $T$ must then coincide with the minimal-time function). If $T$ fails to satisfy (5), then our conjecture is certainly false (and the way in which (5) fails may help us amend it).

We now rain on this parade by pointing out that there are serious obstacles to rigorously justifying the route that we have just outlined. There is, to begin with, the issue of controllability: Is it always possible to steer $\alpha$ to 0 in finite time? And if this holds, do minimal-time trajectories exist? Even if this is true, how do we know that $T(\cdot)$ is differentiable? If this fails to be the case, then we shall need to replace the gradient $\nabla T$ used above by some suitably generalized derivative. Next, we would have to examine anew the argument that led to the HamiltonJacobi equation (5), which itself will require reformulation in some way that allows for nonsmooth solutions. Will the Hamilton-Jacobi equation generalized in such a way admit $T$ as the unique solution?

Assuming that all this can be done, the second stage above offers fresh difficulties of its own. Even if $T$ is smooth, there is in general no continuous function $k(\cdot)$ satisfying $(6)$ for each $x$. When $k$ is discontinuous, the classical concept of 'solution' to (7) is inappropriate; what solution concept should we use instead? Would optimal trajectories still result?

That these difficulties are real, and indeed that they arise in the simplest problems, can be illustrated by the following example, familiar from any introductory text in optimal control.

The double integrator. This refers to the system $x^{\prime \prime}=u$, or, in terms of the standard formulation $(*)$ :

$$
x^{\prime}(t)=y(t), y^{\prime}(t)=u(t), u(t) \in[-1,+1] .
$$

Thus $n=2, m=1$, and the dynamics are linear. It is not difficult to show that all initial points $(x(0), y(0))=(\alpha, \beta)$ are controllable to the origin in finite time; existence theory tells us that minimal-time trajectories exist. The Maximum Principle (see Section 2) helps us to identify them: they turn out to be bang-bang with at most one switch between +1 and -1 . We can then calculate the minimal-time function $T(\cdot)$ :

$T(\alpha, \beta)= \begin{cases}-\beta+\sqrt{2 \beta^{2}-4 \alpha} & \text { when }(\alpha, \beta) \text { is left of } S \\ +\beta+\sqrt{2 \beta^{2}+4 \alpha} & \text { when }(\alpha, \beta) \text { is right of } S\end{cases}$

where the switching curve $S$ in the $x-y$ plane is given by $y^{2}=2|x|$; see Fig. 1. The resulting function $T(\cdot)$ is seen to be continuous, but it fails to be differentiable or even locally Lipschitz along the switching curve. The optimal feedback synthesis consists of taking $k=-1$ to the right or on the upper branch) of $S$, and $k=+1$ otherwise.

We see therefore that our doubts correspond to real difficulties, and they explain why the dynamic programming approach to optimal control, very prominent in the $1950 \mathrm{~s}$ and $60 \mathrm{~s}$, is now frequently ignored in engineering texts, or else relegated to a heuristic role, perhaps in exercises. In fact, however, the difficulties have now been successfully and rigorously resolved, through the use of nonsmooth analysis, viscosity solutions, and discontinuous feedbacks. 


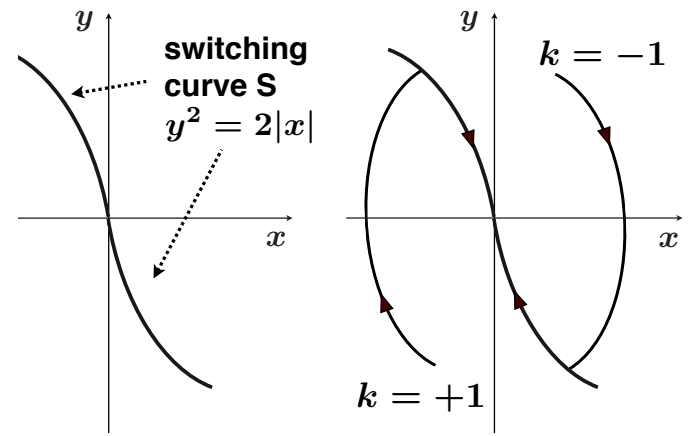

Fig. 1. The double integrator: switching curve and optimal synthesis

These very same tools will play a central role in the stabilization issue, which we turn to now.

\section{Controllability and stabilization}

We consider anew the system $(*)$. We still wish to guide the state $x$ to the origin, but there is no particular desire to minimize the time required to do so, nor any other criterion; a 'reasonable' stabilization method suffices. It is natural to hope that the technical difficulties encountered above can be avoided, since optimality is not an issue.

The key to driving the state to the origin resides in the design of a suitable feedback $k$, by which we mean simply a function on $\mathbb{R}^{n}$ having values in $U$. Our goal is the following: to find a feedback $k$ for which the resulting ordinary differential equation

$$
x^{\prime}=g(x):=f(x, k(x))
$$

is asymptotically stable. Precise definitions will be given later; for now let us just say that stability implies that solutions $x(\cdot)$ of $(9)$ converge to 0 as $t \rightarrow+\infty$.

There is on hand a tool for analyzing this property introduced by Lyapunov over a century ago, and wellknown to engineers. It involves the existence of a (smooth) function $V: \mathbb{R}^{n} \rightarrow \mathbb{R}$, now known as a Lyapunov function, having certain properties:

Positive definiteness: $\quad V(0)=0$ and $V(x)>0 \quad \forall x \neq 0$.

Properness: The level sets $\{x: V(x) \leq c\}$ are compact for every $c$; equivalently, $V$ is radially unbounded:

$$
V(x) \rightarrow+\infty \text { as }|x| \rightarrow+\infty .
$$

Infinitesimal decrease:

$$
\langle\nabla V(x), g(x)\rangle<0 \quad \forall x \neq 0 .
$$

The celebrated theorem of Lyapunov states that if such a function $V$ exists, then the differential equation $x^{\prime}=g(x)$ is stable.

Later, in the classical works of Massera, Barbashin and Krasovskii, and Kurzweil, this sufficient condition for stability was also shown to be necessary (under various sets of hypotheses). Such results are known as converse Lyapunov theorems. Thus, if the differential equation $x^{\prime}=$ $g(x)$ is stable, then there exists a Lyapunov function $V$ associated with $g$.
The application of these classical results to control systems would work as follows. Suppose first that we have found a feedback $k$ so that the resulting differential equation (9) is stable. Then a classical Lyapunov function $V$ exists for $g$, and it follows that the system $(*)$ admits a controlLyapunov function: a (smooth) positive definite, proper function $V$ satisfying:

$$
h(x, \nabla V(x))=\min _{u \in U}\langle\nabla V(x), f(x, u)\rangle<0 \quad \forall x \neq 0,
$$

where $h$ is defined as before by (4).

Conversely, if we have a function $V$ satisfying (11), then $V$ leads to a suitable feedback: simply define $k(x)$, for any $x \neq 0$, to be any point $u$ in $U$ for which

$$
\langle\nabla V(x), f(x, u)\rangle<0 .
$$

Then $V$ is a classical Lyapunov function for (9); by Lyapunov's criterion, we deduce that (9) is asymptotically stable.

These arguments, which may be found as given above in certain recent engineering texts, seem to establish two things: first, that the possibility of stabilizing the system by some feedback $k$ is equivalent to the existence of a control-Lyapunov function $V$; secondly, that a stabilizing feedback can be designed on the basis of such a function, in the manner indicated above.

Note the analogy with the two stages of the dynamic programming approach described earlier (compare for example (3) and (11)): finding a control-Lyapunov function $V$ is analogous to identifying the minimal-time function $T(\cdot)$, and in each case the function is used to construct an appropriate feedback $k$. Unfortunately, the analogy is a very good one: it goes further, in that the obstacles to the dynamic programming approach (based upon regularity considerations for $T$ and $k$ ) carry over with full force to the stabilization context.

This may be surprising, for three reasons. First, as mentioned above, stability and the existence of a smooth Lyapunov function are indeed equivalent in the case of a single (uncontrolled) ordinary differential equation. Second, further circumstantial evidence of a positive nature is provided by the case of linear systems with unconstrained controls (see below), in which controllability and feedback stabilizability are virtually synonyms. Third, although minimal-time functions are nonsmooth, we now seek merely a 'reasonable' convergence to 0 ; this would appear to be much less demanding, and our intuition may tell us that a smooth Lyapunov function should exist (assuming of course that the system is controllable).

It turns out that both the positive evidence and our intuition are quite misleading. As we shall see, the stability of nonlinear systems cannot be reduced to continuous feedbacks and smooth control-Lyapunov functions.

As regards the feedback definition aspect, the basic difficulty is the same: we cannot in general make a continuous selection of a function $k$ satisfying

$$
\langle\nabla V(x), f(x, k(x))\rangle<0,
$$

even if a smooth control-Lyapunov function $V$ is available. This is quite clear in general: just consider the case in 
which $U$ consists of finitely many points (even for a linear system). But the impossibility also manifests itself even when $U=\mathbb{R}^{m}$.

As regards the existence of a smooth $V$, the issue is more subtle. The argument given above does establish that when a continuous stabilizing feedback $k$ exists, then it induces a smooth control-Lyapunov function. But such a continuous $k$ may not exist; many (including the author) believe that this is typically the case for controllable nonlinear systems. And when a continuous $k$ fails to exist, then the system may or may not admit a smooth control-Lyapunov function (as we shall see).

As in the case of dynamic programming, these regularity difficulties are fundamental, real, and widespread (in the nonlinear setting, or when control values are constrained). Unless control-Lyapunov functions are to be relegated to merely heuristic status (as happened to dynamic programming), an underlying theory that allows a rigorous treatment of these regularity issues must be developed.

\section{The linear case, linearization}

The classical and most familiar context in which engineers encounter system $(*)$ occurs in linear systems theory, the special case of $(*)$ in which

$$
f(x, u)=A x+B u, U=\mathbb{R}^{m} .
$$

(Alternatively, $U$ is taken to be a 'sufficiently large' neighborhood of 0 , for local analysis.) In this setting, controllability is equivalent to the existence of a smooth (in fact, linear) stabilizing feedback, and a smooth (in fact, quadratic) Lyapunov function is available. Despite the central role of such systems in the literature, very few systems of real interest are in fact linear; yet, linear systems theory accounts for the vast majority of the many spectacular contributions to our technology made by control engineers. The explanation of this apparent paradox lies in the use of the principal tool of classical applied mathematics: linearization.

In order to study the nonlinear system $(*)$ in a neighborhood of the equilibrium $(x, u)=(0,0)$ (say), we use the linearized system (12) in which $A:=D_{x} f(0,0)$ and $B:=D_{u} f(0,0)$ are the usual Jacobian matrices. Then, under suitable conditions, since $f(x, u) \cong A x+B u$ for small $(x, u)$, we may reasonably expect the local system properties and feedbacks of the linearized system to carry over to the original one; classical tools such as the implicit function theorem are used in the analysis.

The requirements of this approach include the following:

- The function $f$ must be differentiable, in order that $A$ and $B$ exist.

- The linear system must be controllable, so that the linearization is useful.

- $(x, u)$ must remain close to $(0,0)$, so that the degree of approximation is adequate.

- $U$ must contain a neighborhood of 0 .

- All values of $x$ near 0 must be admissible.
These elements are often present, of course, but not always. There are interesting systems in which $f$ is nondifferentiable, for example when friction, diodes, or other directional phenomena are present (see for example Clarke (1983), Brogliato (1999), and Orlov (2009)). The linear approximation of a real mechanical system can fail to be controllable (as in the nonholonomic integrator, see below). Disturbances may be too large for $(x, u)$ to remain suitably close to $(0,0)$. In certain problems, the control set does not contain a neighborhood of 0 , and there may be constraints on the state from the underlying model (for example, that $x(t)$ must remain in the positive orthant).

Our interest lies in nonlinear systems for which, for whatever reason, linearization is inappropriate. We turn now to a review of some results on that topic.

\section{Strongly stable systems and Lyapunov functions}

There are two natural ways to extend to control systems the concept of asymptotic stability of an ordinary differential equation (ode). They involve either requiring that all, or else some trajectories are attracted to the origin.

We consider now the first alternative, by defining strong Lyapunov stability at the origin as follows: given any $\epsilon>0$, there exists $\delta>0$ such that, for every trajectory $x(\cdot)$ having $x(0) \in B(0, \delta)$, we have $x(t) \in B(0, \epsilon) \forall t \geq 0$. The system $(*)$ is said to be strongly asymptotically stable if it satisfies strong Lyapunov stability at the origin together with the following property: every trajectory $x(\cdot)$ is defined on $[0,+\infty)$ and satisfies $x(t) \rightarrow 0$ as $t \rightarrow+\infty$.

The natural context for the next result is that of a differential inclusion (see Section 2):

$$
x^{\prime}(t) \in F(x(t)) \text { a.e., }
$$

which is more general than the system $(*)$ (just take $F(x):=f(x, U))$. Notice that the definition of strong asymptotic stability given above applies to a differential inclusion without any changes (since only trajectory properties are involved).

The property of strong asymptotic stability of differential inclusions, or of control systems like $(*)$, is in fact characterized by the existence of a smooth Lyapunov function, as the following result shows. It includes as a special case that of an ode $x^{\prime}=g(x)$ in which $g$ is merely continuous (by taking $F(x)=\{g(x)\}$ ). For this reason, it unifies and extends the classical converse Lyapunov theorems (notably that of Massera and Kurzweil).

Theorem 1. (Clarke, Ledyaev, Stern 1998). Let the multifunction $F$ have compact convex values and closed graph. Then (13) is strongly asymptotically stable if and only if there exists a $C^{\infty}$ function $V: \mathbb{R}^{n} \rightarrow \mathbb{R}$ which is positive definite and proper and satisfies

$$
\max _{v \in F(x)}\langle\nabla V(x), v\rangle<0 \quad \forall x \neq 0 .
$$

We refer to such a function $V$ as a strong control-Lyapunov function for the system; note that 'max' has replaced 'min' in (11). In this result, the system multifunction $F$ itself need not even be continuous, yet strong stability is equivalent to the existence of a smooth Lyapunov function: this is a surprising aspect of the theorem. It stands in 
sharp contrast to the case of weak stability (or controllability), where even under much stronger hypotheses on the underlying system, Lyapunov functions will need to be nondifferentiable.

While strong stability has several uses (as in analyzing perturbations of ode's or stability of Filippov solutions), it it weak stability, better known as controllability, that is the relevant concept for our purposes; it is our next topic.

Controllable systems and Lyapunov functions

The following definitions extend in a natural way to control systems the familiar concept of uniform asymptotic stability of ordinary differential equations, with a controllability component.

The system $(*)$ is said to satisfy weak Lyapunov stability at the origin provided that, given any $\epsilon>0$, there exists $\delta>0$ such that, for every $\alpha \in B(0, \delta)$, there is a control $u(\cdot)$ and a corresponding trajectory $x(\cdot)$ defined on $[0,+\infty)$ having $x(0)=\alpha$ and satisfying $x(t) \in B(0, \epsilon) \quad \forall t \geq 0$.

The system $(*)$ is said to be asymptotically controllable (to the origin) if it satisfies local Lyapunov stability together with the following property: every $\alpha \in \mathbb{R}^{n}$ admits a control $u(\cdot)$ and corresponding trajectory $x(\cdot)$ defined on $[0,+\infty)$ having $x(0)=\alpha$ and such that $x(t) \rightarrow 0$ as $t \rightarrow+\infty$. (To contrast with strong stability, this could be called weak stability; we adhere, however, to the standard terminology.)

The appropriate definition of Lyapunov function corresponding to this concept is referred to as a (smooth) control-Lyapunov function (clf) for the system (*): a continuously differentiable, positive definite, proper function $V$ that satisfies (11). Notice that 'min' has replaced the 'max' of Theorem 1 in the weaker form of the infinitesimal decrease condition that figures here.

It is a fact that if the system admits a smooth clf, then it is asymptotically controllable. We now pose the evident question: Is there always a smooth clf to confirm asymptotic controllability? That is, do the familiar converse Lyapunov theorems of Massera and Kurzweil extend to the controllability setting? The answer is decidedly negative. Let us establish this with the help of the following result, in which 'co' refers to 'convex hull': co $S$ is the smallest convex set containing $S$.

Theorem 2. (Clarke, Ledyaev, Stern 1998). Let the system $(*)$ admit a smooth control-Lyapunov function. Then for every $\delta>0$, the following set is a neighborhood of 0 :

$$
\{v \in \operatorname{cof}(x, U): x \in B(0, \delta)\} .
$$

The covering condition that appears in the theorem is therefore a necessary condition for the existence of a clf; note that the sufficiency is not asserted. The theorem allows us to conclude that when the covering condition fails, then no clf exists; the following is an illustration.

The nonholonomic integrator. This term refers to the following well-known system:

$$
x_{1}^{\prime}=u_{1}, x_{2}^{\prime}=u_{2}, x_{3}^{\prime}=x_{1} u_{2}-x_{2} u_{1},
$$

for which $n=3$ and $m=2 ; U$ is taken to be the closed unit ball in $\mathbb{R}^{2}$. It is the simplest representative of a certain class of systems arising in mechanics (see for example the discussion in Sontag (1999)). It would seem to be as wellstructured a system as could be hoped for, within the class of regrettably nonlinear ones: it is linear (separately) in the state and in the control variables, and the control set is ample. We remark that the system is easily seen to be asymptotically controllable. Observe, however, that the covering condition of the theorem fails: for no $x$ is there a point in $\operatorname{co} f(x, U)=f(x, U)$ of the form $(0,0, \gamma)$ where $\gamma \neq 0$. It follows from the theorem that this system does not admit a smooth control-Lyapunov function.

\section{Control-Lyapunov functions in the Dini sense}

In order to address the evident gap between asymptotic controllability and the existence of a clf, we can try to weaken the concept of clf so that it remains sufficient for asymptotic controllability, while also being necessary. A seminal result due to Sontag does just that, using a classical notion introduced by Dini in the 19th century.

Given a function $\phi: \mathbb{R}^{n} \rightarrow \mathbb{R}$, the notation $d \phi(x ; v)$ refers to the (lower) Dini derivate at $x$ in the direction $v$ :

$$
d \phi(x ; v):=\liminf _{t \downarrow 0, w \rightarrow v} \frac{\phi(x+t w)-\phi(x)}{t} .
$$

We remark that if $\phi$ is Lipschitz in a neighborhood of $x$, then $d \phi(x ; v)$ coincides with the simpler expression

$$
\liminf _{t \downarrow 0} \frac{\phi(x+t v)-\phi(x)}{t} .
$$

If $\phi$ is continuously differentiable near $x$, then

$$
d \phi(x ; v)=\langle\nabla \phi(x), v\rangle=\phi^{\prime}(x ; v),
$$

where $\phi^{\prime}(x ; v)$ is the usual directional derivative.

A control-Lyapunov function in the Dini sense (Dini clf) is a continuous function $V$ satisfying properness and positive definiteness, as well as the following derivate variant of infinitesimal decrease (cf. (11)):

$$
\min _{u \in U} d V(x ; f(x, u))<-W(x) \quad \forall x \neq 0 .
$$

(The minimum is attained because the function $v \mapsto$ $d V(x ; v)$ is lower semicontinuous.)

Here, $W: \mathbb{R}^{n} \backslash\{0\} \rightarrow(0,+\infty)$ is a rate function, which simply means that $W$ is bounded away from 0 on any compact subset of $\mathbb{R}^{n} \backslash\{0\}$. When $V$ is a $C^{1}$ clf, it is easy to see that the existence of such a rate function $W$ is automatic (whereas it must be postulated for a Dini clf). Thus a smooth clf in the earlier sense is also a Dini clf. And of course a Dini clf which is also continuously differentiable is a smooth clf. We remark that without loss of generality, and in contrast to $V$, the rate function $W$ can always be assumed to be continuously differentiable on $\mathbb{R}^{n} \backslash\{0\}$ if desired (Clarke et al., 1998, p. 211).

Extended to nonsmooth functions in this way, clf's now characterize asymptotic controllability:

Theorem 3. (Sontag 1983). The system (*) is asymptotically controllable if and only if there exists a controlLyapunov function in the Dini sense. 


\section{Stabilizing feedbacks}

We turn now to the feedback stabilization issue. If there exists a continuous feedback $k(x)$ such that the ensuing differential equation

$$
x^{\prime}(t)=g(x(t)), \quad \text { where } \quad g(x):=f(x, k(x))
$$

is asymptotically stable, then it is easy to see that the system is asymptotically controllable. (The open-loop control $u(t):=k(x(t))$ drives $x$ to 0 .) A central question in the subject has long been the converse: If the system is asymptotically controllable to the origin, is there a feedback $k$ such that the resulting $g$ exhibits global asymptotic stability?

It is of course quite possible for a system featuring a finite discrete control set to be asymptotically controllable: consider, for example, the double integrator of Section 1 , with $U$ redefined to be $\{-1,+1\}$. For such a system, the only continuous feedbacks are constant. Thus, in full generality, it is clear that not every asymptotically controllable system will admit a continuous stabilizing feedback. The question of the converse, then, is to be interpreted as bearing upon certain classes of 'reasonable' or 'nice' systems, which is usually interpreted to mean systems which are affine in the control and have a control set which is a neighborhood of 0 .

There is a natural preference for continuous feedbacks $k$, for several reasons. The most basic one stems from the desire that the resulting function $g(x):=f(x, k(x))$ be continuous, for this is the minimal requirement of the classical theory of ordinary differential equations. In its absence, we would not even have a clear idea of what a solution of $x^{\prime}=g(x)$ is supposed to mean. (Another reason for favoring continuous feedback arises from robustness considerations, which we discuss later.)

The following celebrated result will shed some light on the question:

Theorem 4. (Brockett 1983). If the system $(*)$ admits a continuous stabilizing feedback, then it has the following covering property: for every $\delta>0$, the set $f(B(0, \delta), U)$ is a neighborhood of 0 .

As we have seen, the nonholonomic integrator fails to satisfy the covering condition of Theorem 2, which is equivalent in this case to that of Theorem 4. It follows, then, that no continuous feedback stabilizes it. As in the case of Theorem 2, we stress that the covering condition is a necessary condition for the existence of a continuous stabilizing feedback, not a sufficient one; this is illustrated by the following example.

Artstein's circles. We consider the following system, in which $n=2$ and $m=1$ :

$$
x_{1}^{\prime}=\left(x_{1}^{2}-x_{2}^{2}\right) u, x_{2}^{\prime}=2 x_{1} x_{2} u, u \in[-1,+1] .
$$

For any point $x \neq 0 \in \mathbb{R}^{2}$, the available velocity vectors $x^{\prime}$ are all tangent to the unique circle in the plane passing through both $x$ and the origin, and centered on the $x_{2}$-axis (see Fig. 2). The case in which $x$ lies on the $x_{1}$-axis may be thought of as corresponding to such a circle having infinite radius: the $x_{1}$-axis itself.
The circle in question is therefore a strongly invariant set (see Theorem 9 below): a trajectory originating on a circle must remain upon it. The direction of movement (clockwise or counterclockwise) depends on the sign of $u$. It is easy to see that the system is asymptotically stable, and that it satisfies the covering condition of Theorems 2 and 4 .

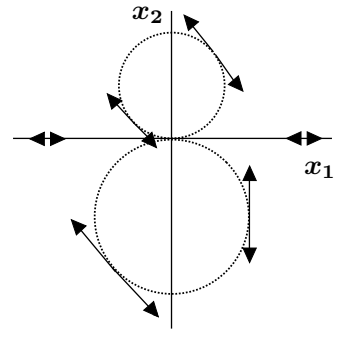

Fig. 2. Artstein's circles
We claim that it admits no stabilizer $k$ which is continuous on $\mathbb{R}^{n} \backslash\{0\}$, which we prove by contradiction. First, we observe that $k(x) \neq$ 0 for all $x \neq 0$, for otherwise $x$ is a nonzero equilibrium of the stabilized system, which cannot be. Next, we observe that $k$ must be negative on the positive $x_{1}$ axis: if it were positive at a point in that set, movement

to the right (away from the origin) would always prevail (since $k$ cannot be 0 ), and the trajectory would not approach the origin. Similarly, $k$ must be positive on the negative $x_{1}$-axis. Now consider any circle in the plane centered at the origin. By continuity, it follows that $k$ vanishes somewhere on the circle. But then the stabilized system has nonzero equilibria: contradiction.

This system also provides an example showing that the covering condition is not sufficient in the context of Theorem 2. For suppose that a smooth clf $V$ did exist, and consider any one of the invariant circles $C$. Then $V$ admits a maximum relative to the compact set $C$ at some point $x$, and $x$ is necessarily nonzero since $V$ is positive definite. We have $\nabla V(x)$ normal to $C$ at $x$, in view of the maximization. But then $\nabla V(x)$ is orthogonal to all available velocities at $x$, and infinitesimal decrease cannot be satisfied at $x$.

A further lesson can be drawn from this example: the nonexistence of a continuous stabilizer cannot be 'approximated away'. That is, even if we were willing to settle for a continuous feedback $k$ that stabilizes (for example) the ball $B(0, R)$ to the ball $B(0, r)$, for $r>0$, the analysis shows that such a $k$ does not exist.

It is possible for the system $(*)$ to be asymptotically stable and admit a smooth clf, yet fail to admit a continuous stabilizer, as we now see.

Example. (Ledyaev and Sontag 1997). Consider the system $x_{1}^{\prime}=u_{2} u_{3}, x_{2}^{\prime}=u_{1} u_{3}, x_{3}^{\prime}=u_{1} u_{2},\left|\left(u_{1}, u_{2}, u_{3}\right)\right| \leq 1$. (17) One may easily check that the function $V=|x|^{2}$ is a smooth clf. The covering condition of Theorem 4 fails to hold, however, since no velocity has the form $(0, \gamma, \kappa)$ for $\gamma \neq 0, \kappa \neq 0$. It follows that no continuous stabilizer exists.

A system which is asymptotically stable can admit both a smooth clf and a continuous stabilizer, or neither, or else a smooth clf but no continuous stabilizer, as we have seen. Thus the two issues are rather decoupled in general. But for certain systems they are equivalent:

Theorem 5. (Artstein 1983). Suppose that the system is affine with respect to $u$, and that $U$ is a compact convex set. Then the system admits a continuous stabilizing feed- 
back if and only if it admits a smooth control-Lyapunov function.

The principal lesson of this rather long introduction is that, in a nonlinear setting, stabilization cannot be reduced to smooth clf's and continuous feedbacks. The recent results in the subject, which have had a somewhat more positive nature than the largely negative ones described above, require much the same tools that successfully resolved the difficulties of the dynamic programming method. These tools are introduced in the following section. Following that, we develop an approach to stabilization which builds upon (and extends) earlier results by Clarke, Ledyaev, Rifford, Sontag, Stern, Subbotin, and Vinter. There is an extensive literature related to the issues discussed here, with important contributions by Ancona, Artstein, Bressan, Brockett, Coron, Freeman, Kellett, Kokotovic, Praly, Prieur, Rosier, Ryan, Sontag, Sussmann, Teel, among many others; we make no pretense to a complete bibliography on the subject.

\section{GENERALIZATIONS OF ODE'S}

Classical solutions. The usual notion of solution of an ode $x^{\prime}=g(x)$, where $g$ is continuous, is of course simply that of a differentiable function $x$ which satisfies, at all points $t$ of the underlying interval $[a, b]$, the condition $x^{\prime}(t)=$ $g(x(t))$. This pointwise criterion actually implies that any such solution $x$ is in fact continuously differentiable $\left(C^{1}\right)$. When $g$ has measurable $t$-dependence (while remaining continuous in $x$ ), the classical solution concept for the ode $x^{\prime}=g(t, x)$ is extended in the manner of Carathéodory: we require that $x$ be absolutely continuous and that the pointwise equality $x^{\prime}(t)=g(t, x(t))$ hold for almost all $t$ in $[a, b]$.

We recall that an absolutely continuous function $x$ may be characterized as a continuous function which admits a representation of the form

$$
x(t)=x(a)+\int_{a}^{t} v(s) d s
$$

for some $v \in L^{1}(a, b)$; in this case we have $x^{\prime}(t)=v(t)$ a.e. It is equivalent to define a Carathéodory solution to be a continuous function $x$ satisfying

$$
x(t)=x(a)+\int_{a}^{t} g(s, x(s)) d s, \quad t \in[a, b] .
$$

The restriction to the class of absolutely continuous functions serves to avoid so-called singular functions $x$ which (for example) can be continuous and strictly increasing, differentiable almost everywhere, but with $x^{\prime}(t)=0$ for almost all $t$. Evidently, functions such as these are not faithfully reflected by their derivatives, and do not lend themselves to the theory of ode's.

In considering the ode $x^{\prime}=g(x)$ with $g$ discontinuous, the first thought that comes to mind is to retain the pointwise criterion; that is, to define a solution to be an absolutely continuous function $x$ such that $x^{\prime}(t)=g(x(t))$ for almost all $t$. This is unsatisfactory, for the simple reason that solutions in this sense generally fail to exist, as we now illustrate.
Example. Consider in one dimension the ode $x^{\prime}=g(x)$ with initial value $x(0)=0$, where

$$
g(x)= \begin{cases}-1 & \text { if } x>0 \\ +1 & \text { if } x \leq 0 .\end{cases}
$$

It is easy to see that no function $x$ satisfies the pointwise condition $x^{\prime}(t)=g(x(t))$ a.e. Yet, intuitively, a consideration of the underlying flow leads us to regard the function $x \equiv 0$ as the 'physically natural' solution. Another approach must be found (we return to this example later).

Differential inclusions. A differential inclusion refers to a multi-valued differential equation of the form

$$
x^{\prime}(t) \in F(x(t)),
$$

where $F$ is a function on $\mathbb{R}^{n}$ whose values are subsets of $\mathbb{R}^{n}$. A solution or trajectory of such a differential inclusion on an interval $[a, b]$ refers to an absolutely continuous function $x:[a, b] \rightarrow \mathbb{R}^{n}$ whose derivative $x^{\prime}(t)$ (which exists almost everywhere, in the sense of Lebesgue measure) belongs for almost $t \in[a, b]$ to the set $F(x(t))$. The graph of $F$ is the set

$$
\{(x, v): v \in F(x)\} .
$$

It is a fact that (under our standing hypotheses), the standard control system $(*)$ is equivalent to the differential inclusion (19) when $F$ is defined by $F(x):=f(x, U)$, in the sense that $(*)$ and $(19)$ admit the same trajectories (this is known as Filippov's Lemma). This fact is useful when we wish to focus upon the trajectories of the system and not the controls that generate them. An example of such a concern is relaxation.

Relaxation, linear growth. The relaxation of the system $(*)$ refers to the differential inclusion

$$
x^{\prime}(t) \in \operatorname{cof}(x(t), U) .
$$

(Thus we avoid speaking of relaxed controls, which are generally taken to be measure-valued.) A relaxed trajectory of $(*)$ means a trajectory of this differential inclusion. We shall say that the system $(*)$ is relaxed if, for every $x$, the set $f(x, U)$ is convex. For the differential inclusion (19), this means that $F$ is convex-valued.

For purposes of stabilization, it is essentially the same to consider either the original (unrelaxed) system or its relaxation. The reason for this is that (see for example (Clarke, 1983, Chap. 3)) any trajectory $y$ of the relaxation can be uniformly approximated (on any bounded interval) to any given tolerance by a trajectory of the unrelaxed system having the same initial value (the Lipschitz behavior in our basic hypotheses is needed here). For this reason it is tempting to consider only relaxed systems, for they have certain features that are convenient, for example as regards existence of solutions to optimal control problems. But we have resisted that temptation.

Another familiar property that plays a role in existence is linear growth: there exist constants $c, k$ such that

$$
v \in F(x) \Longrightarrow|v| \leq c|x|+k .
$$

This condition serves to preclude finite blow-up of trajectories (via an estimate obtained by Gronwall's Lemma). It is often superfluous in the context of stabilization, notably 
when the state is guaranteed to remain bounded because it evolves in a level set of a Lyapunov function.

Filippov solutions. The best known solution concept for the ode $x^{\prime}=g(x)$ when $g$ is discontinuous is that of Filippov. An absolutely continuous function $x$ is said to be a Filippov solution of $x^{\prime}(t)=g(x(t))$ provided that it satisfies the differential inclusion

$$
x^{\prime}(t) \in G(x(t)) \text { a.e. }
$$

where the multivalued function $G$ is defined by

$$
G(x):=\bigcap_{\delta>0} \bigcap_{\text {meas } \Omega=0} \overline{\operatorname{co}} g(B(x, \delta) \backslash \Omega) .
$$

The second intersection is taken over all sets $\Omega$ of measure 0 ; its purpose is to take into account only 'essential' values of $g$. It is easy to see that $G(x)$ reduces to $\{g(x)\}$ if $g$ is continuous at $x$.

Let us illustrate this in the case of example (18). We calculate

$$
G(x)= \begin{cases}\{-1\} & \text { if } x>0 \\ {[-1,+1]} & \text { if } x=0 \\ \{+1\} & \text { if } x<0\end{cases}
$$

It follows that the unique Filippov solution of the initialvalue problem $x^{\prime}=g(x), x(0)=0$, is the natural one: $x \equiv 0$.

This example provides insight into the reason behind the presence of the convex hull operation in the Filippov definition: it is there to provide existence of a solution. The resulting solution concept has several other positive features. It can lead, however, to 'too many solutions', a fact that we proceed to illustrate.

Example. A discontinuous vector field in the $x$ - $y$ plane having only three different values is implicitly defined by Fig. 3. (At points of discontinuity, one of the 'nearby' arrows may be assigned, in any desired fashion.) It is clear that, from the initial condition $(0,0)$, there are two natural solutions, both moving to the right: one along the line $y=x$, the other along $y=-x$. But if we apply the Filippov definition, then (because of the convex hull operation) we find many other solutions; for example, one that moves to the right along the positive $x$-axis.

It is the existence of such superfluous trajectories that explains why Filippov solutions are inappropriate for feedback stabilization, as we shall see in Section 7.

We turn now to a solution concept that is better suited to our purposes, and we limit attention to the case that really concerns us: the implementation in the system $(*)$

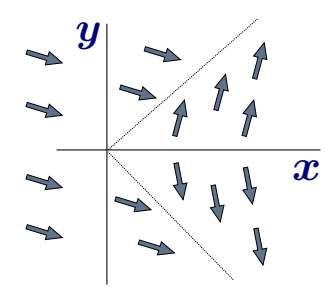

Fig. 3. A vector field of a possibly discontinuous feedback $k$.

Sample-and-hold solutions. This solution concept begins with a partition $\pi=\left\{t_{i}\right\}_{i \geq 0}$ of $[0, \infty)$, by which we mean a countable, strictly increasing sequence $t_{i}$ with $t_{0}=0$ such that $t_{i} \rightarrow+\infty$ as $i \rightarrow \infty$. The diameter of $\pi$, denoted $\operatorname{diam}(\pi)$, is defined as $\sup _{i \geq 0}\left(t_{i+1}-t_{i}\right)$.

Now let there be given an initial condition $x_{0}$, a feedback $k$, and a partition $\pi$. A corresponding $\pi$-trajectory refers to an absolutely continuous function $x:[0,+\infty) \rightarrow \mathbb{R}^{n}$ such that, for each $i \geq 0$, we have

$$
x^{\prime}(t)=f\left(x(t), k\left(x_{i}\right)\right), \quad x\left(t_{i}\right)=x_{i}, \quad t_{i} \leq t \leq t_{i+1},
$$

where $x_{i}:=x\left(t_{i}\right)$ are the so-called nodes of $x$.

Less formally, this corresponds to constructing $x$ in stepby-step fashion as follows: Between $t_{0}$ and $t_{1}$, we consider a classical solution $x$ of the ode

$$
x^{\prime}(t)=f\left(x(t), k\left(x_{0}\right)\right), \quad x(0)=x_{0}, \quad t_{0} \leq t \leq t_{1} .
$$

At most one such solution can exist, since $f$ is locally Lipschitz in $x$. Later, finite-time blow-up will be precluded because $x(t)$ remains in a bounded level set of a clf. The consequence of these observations is that a unique solution $x$ exists. We then set $x_{1}:=x\left(t_{1}\right)$, and we consider the following ode on the next partition interval $\left[t_{1}, t_{2}\right]$ :

$$
x^{\prime}(t)=f\left(x(t), k\left(x_{1}\right)\right), \quad x\left(t_{1}\right)=x_{1}, \quad t_{1} \leq t \leq t_{2},
$$

in which the constant control value has been updated to $k\left(x_{1}\right)$. And so on in this fashion. We sometimes write $x_{\pi}$ for $x$, since the (real) trajectory obtained above evidently depends on the partition $\pi$. Note that no regularity is asked here of $k$; it suffices that it be defined.

A limiting sample-and-hold trajectory (for the given feedback) refers to any function $x$ which, on any bounded interval, is obtainable as the uniform limit of a sequence $x_{\pi_{j}}$ of such $\pi$-trajectories for which the underlying partitions $\pi_{j}$ satisfy diam $\pi_{j} \rightarrow 0$. When linear growth is imposed (we shall not do so), it follows that the set of limiting sample-and-hold trajectories from a given initial condition is nonempty. When the system $(*)$ is relaxed, any such $x$ is an actual trajectory of $(*)$.

It is not hard to see that in the example (18), as well as in that of Fig. 3, the limiting sample-and-hold solutions for the given initial conditions are precisely the natural ones.

\section{NONSMOOTH ANALYSIS}

We now present a few basic definitions and facts in nonsmooth analysis. A complete treatment is given in the book Clarke et al. (1998) (which has been accused of readability).

\section{Generalized gradients}

A function $f: \mathbb{R}^{n} \rightarrow \mathbb{R}$ is said to be Lipschitz on a set $S \subset \mathbb{R}^{n}$ if there exists $K \geq 0$ such that, for all $x, y \in S$,

$$
|f(y)-f(x)| \leq K|y-x| .
$$

We say that $f$ is locally Lipschitz on $S$ if each point $z \in S$ admits a radius $r>0$ and a constant $K$ (both depending on $z$ ) such that the Lipschitz condition (21) holds for all $x, y \in B(z, r)$. This is equivalent to requiring that the Lipschitz condition hold on any bounded subset $S^{\prime}$ of $S$ (for some $K$ depending on $S^{\prime}$ ). 
The Lipschitz property is closed under many operations, such as sums, lower or upper envelopes, compositions, etc. It is a fundamental result in analysis that a function which is Lipschitz on an open set in $\mathbb{R}^{n}$ is differentiable almost everywhere in the set (Rademacher's Theorem).

When $f$ is Lipschitz at a point $x$ (that is, on a neighborhood of $x$ ), the generalized gradient $\partial_{C} f(x)$ of $f$ at $x$ is given by

$$
\partial_{C} f(x)=\operatorname{co}\left\{\lim _{i \rightarrow \infty} \nabla f\left(x_{i}\right): x_{i} \rightarrow x, x_{i} \notin \Omega\right\} .
$$

Here 'co' denotes 'convex hull', $\Omega$ is any set of measure zero, and $x_{i}$ is any sequence converging to $x$ while avoiding $\Omega$, and along which $\nabla f\left(x_{i}\right)$ exists and converges. Because $f$ is differentiable almost everywhere near $x$, there exist many such sequences.

It is a fact that $\partial_{C} f(x)$ is a compact convex set which is independent of the choice of $\Omega$. Clearly, we have

$$
\partial_{C} f(x)=\{\nabla f(x)\}
$$

if $f$ is continuously differentiable in a neighborhood of $x$.

We proceed to list a few facts in the calculus of generalized gradients. Recall that for a convex function $f$, the subdifferential in the sense of convex analysis, denoted $\partial f(x)$, is defined as follows:

$$
\zeta \in \partial f(x) \Longleftrightarrow f(y)-f(x) \geq\langle\zeta, y-x\rangle \forall y .
$$

Theorem 6. (Properties of generalized gradients).

1. If $f$ is convex, then $\partial_{C} f(x)=\partial f(x)$.

2. If $\left\{f_{i}\right\}$ is a finite collection of functions each of which is $C^{1}$ near $x$, and if $f:=\max _{i} f_{i}$ is the upper envelope of the family, then

$$
\partial_{C} f(x)=\operatorname{co}\left\{\nabla f_{i}(x): i \in I(x)\right\},
$$

where $I(x)$ is the set of indices $i$ for which $f(x)=f_{i}(x)$.

3. If $f$ and $g$ are Lipschitz at $x$, then

$$
\partial_{C}(f+g)(x) \subset \partial_{C} f(x)+\partial_{C} g(x) .
$$

4. If $f$ is Lipschitz at $x$, if $\zeta_{i} \in \partial_{C} f\left(x_{i}\right)$, where $\zeta_{i} \rightarrow \zeta$ and $x_{i} \rightarrow x$, then $\zeta \in \partial_{C} f(x)$.

Other calculus facts (such as the chain rule, product rule, mean value theorem, Lagrange multiplier rule) also play a role in calculating and using generalized gradients, whose theory also includes geometrical constructs (tangent and normal vectors), as well as an extension to vector-valued functions (and corresponding nonsmooth versions of the implicit and inverse function theorems).

Example. We define a Lipschitz function $f$ on $\mathbb{R}^{2}$ by

$$
f(x, y)=\max \{\min [x,-y], y-x\} .
$$

Then

$$
f(x, y)=\left\{\begin{aligned}
x & \text { if } x \in A \\
-y & \text { if } x \in B \\
y-x & \text { if } x \in C
\end{aligned}\right.
$$

where the regions $A, B, C$ are as indicated in Fig. 4.

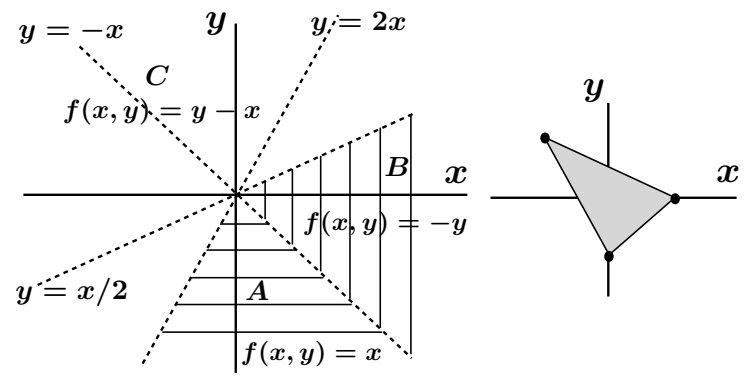

Fig. 4. A function $f$ and its generalized gradient at 0

We calculate $\partial_{C} f(0,0)$ via (22), ignoring the points that lie in the boundaries of $A, B$, or $C$ (these form a set of zero measure). Then the gradient values $(1,0),(0,-1)$, and $(-1,1)$ are involved, and we find

$$
\partial_{C} f(0,0)=\operatorname{co}\{(1,0),(0,-1),(-1,1)\} .
$$

The maximum principle

The earliest use of generalized gradients in control occurred in the formulation and proof of general (and in particular, nonsmooth) versions of the Pontryagin Maximum Principle; see Clarke (1983). This is a set of necessary conditions which must be satisfied by optimal controls and trajectories, and which helps to identify them.

Let us illustrate this in the context of minimizing the cost

$$
J(x, u):=\int_{0}^{T} L(x(t), u(t)) d t
$$

relative to the controls $u$ and corresponding trajectories $x$ satisfying $(*)$ together with an initial condition $x(0)=x_{0}$. Here $L$ is taken to be locally Lipschitz.

The Pontryagin Hamiltonian $\mathcal{H}$ is defined as follows:

$$
\mathcal{H}(x, p, u):=\langle p, f(x, u)\rangle-L(x, u) .
$$

Theorem \%. If $(x, u)$ solves this problem, then there exists an absolutely continuous function $p$ such that $p(T)=0$ and, for almost all $t \in[0, T]$,

$$
\begin{aligned}
-p^{\prime}(t) & \in \partial_{C}\{\mathcal{H}(\cdot, p(t), u(t))\}(x(t)), \\
u(t) & \in \operatorname{argmax}_{u \in U} \mathcal{H}(x(t), p(t), u) .
\end{aligned}
$$

(Notation: $\operatorname{argmax}_{v \in V} g(v)$ is the set of points in $V$ at which $g$ attains a maximum.)

In the above, $T$ can be either prescribed, or else free to vary under some constraints; in the latter case, further conclusions bearing upon $T$ and $p(T)$ are asserted.

There exist many types of problems involving additional dynamic or boundary constraints: see Clarke (2005), Vinter (2000), and the references therein. The maximum principle has also been extended to hybrid optimal control problems: see Clarke and Vinter (1989).

\section{Proximal subgradients}

We turn now to a different (but related) type of generalized derivative. 
An extended-valued function $\phi: \mathbb{R}^{n} \rightarrow \mathbb{R}^{+\infty}:=\mathbb{R} \cup\{+\infty\}$ is one which can take the value $+\infty$ at some points. The effective domain of $\phi$, denoted $\operatorname{dom} \phi$, refers to the set of points $x$ for which $\phi(x)<\infty$. The function is said to be lower semicontinuous if, at each $x$, for any sequence $x_{i}$ converging to $x$ for which $\lim _{i} \phi\left(x_{i}\right)$ exists (finite or not), we have $\phi(x) \leq \lim _{i} \phi\left(x_{i}\right)$. This class of functions is the natural context for the theory of proximal subgradients, which we proceed to describe briefly.

A vector $\zeta \in \mathbb{R}^{n}$ is said to be a proximal subgradient of $\phi$ at $x \in \operatorname{dom} \phi$ provided that, for some $\sigma \geq 0$ and some neighborhood $N_{x}$ of $x$, we have

$$
\phi(y)-\phi(x)+\sigma|y-x|^{2} \geq\langle\zeta, y-x\rangle \quad \forall y \in N_{x} .
$$

The set of such $\zeta$ is called the proximal subdifferential of $\phi$ at $x$, denoted $\partial_{P} \phi(x)$.

In contrast to the generalized gradient, the proximal subdifferential can be empty at certain (even many) points, a feature which, perversely, turns out to be one of its charms.

If $\phi$ is differentiable at $x$, then $\partial_{P} \phi(x)$ is either the singleton set $\{D \phi(x)\}$ or else the empty set. If $\phi$ is $C^{2}$ in a neighborhood of $x$, then $\partial_{P} \phi(x)=\{D \phi(x)\}$. When $\phi$ is convex, then $\partial_{P} \phi(x)$ coincides with the subdifferential of convex analysis.

The geometric content of (23) is that $f$ admits a parabola which contacts the graph of $f$ at the point $(x, f(x))$ while remaining below the graph locally. Then the slope of the parabola at $x$ defines a proximal subgradient.

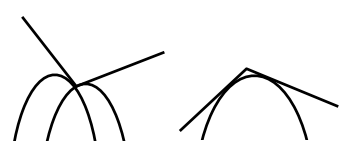
parabolas, and none
Fig. 5. Many supporting

There may be many such 'contact parabolas', or none. If the function is sufficiently smooth, all contact parabolas will have the same slope at $x$; this will then define the unique proximal subgradient. In Fig. 5 we see a function which has a "convex corner' at a point $x$, and another function having a 'concave corner' at $x$. The proximal subdifferentials at $x$ are, respectively, $[-2,1]$ (say) and the empty set.

The calculus of proximal subgradients is complete but 'fuzzy'. To give but one example, consider the sum rule. There is no hope for a general formula such as

$$
\partial_{P}\left(\phi_{1}+\phi_{2}\right)(x)=(\text { or } \subset) \partial_{P} \phi_{1}(x)+\partial_{P} \phi_{2}(x) .
$$

Instead, it takes the following form: if $\zeta \in \partial_{P}\left(\phi_{1}+\phi_{2}\right)(x)$, then for any $\epsilon>0$ there exist $x_{1}, x_{2} \in B(x, \epsilon)$ such that

$$
\zeta \in \partial_{P} \phi_{1}\left(x_{1}\right)+\partial_{P} \phi_{2}\left(x_{2}\right)+B(0, \epsilon) .
$$

Proximal calculus includes useful geometrical constructs. Given a nonempty closed subset $S$ of $\mathbb{R}^{n}$ and a point $x$ in $S$, we say that $\zeta \in \mathbb{R}^{n}$ is a proximal normal (vector) to $S$ at $x$ if there exists $\sigma=\sigma(x, \zeta) \geq 0$ such that

$$
\langle\zeta, y-x\rangle \leq \sigma|y-x|^{2} \forall y \in S .
$$

This is the proximal normal inequality. The set of such $\zeta$ which is a convex cone containing 0 , is denoted $N_{S}^{P}(x)$ and is referred to as the proximal normal cone.
For a given set $S$, the notation $I_{S}$ refers to the indicator function of $S$ : the function whose value on $S$ is 0 and whose value is $+\infty$ elsewhere. Then, for any $x \in S$, we have

$$
\partial_{P} I_{S}(x)=N_{S}^{P}(x) \text {. }
$$

The limiting normal cone $N_{S}^{L}(x)$ to $S$ at $x$ is obtained by applying a sequential closure operation to $N_{S}^{P}$ :

$$
N_{S}^{L}(x):=\left\{\lim \zeta_{i}: \zeta_{i} \in N_{S}^{P}\left(x_{i}\right), x_{i} \rightarrow x, x_{i} \in S\right\} .
$$

If the set $S$ is convex, the two normal cones defined above coincide with the familiar normal cone of convex analysis, in which $\zeta$ is normal to $S$ at $x$ iff

$$
\left\langle\zeta, x^{\prime}-x\right\rangle \leq 0 \forall x^{\prime} \in S .
$$

If $S$ is a smooth manifold, or manifold with boundary, they coincide with the classical normal space, or half-space.

A similar limiting procedure defines the limiting subdifferential:

$\partial_{L} \phi(x)=\left\{\lim \zeta_{i}: \zeta_{i} \in \partial_{P} \phi\left(x_{i}\right), x_{i} \rightarrow x, \phi\left(x_{i}\right) \rightarrow \phi(x)\right\}$.

When $\phi$ is locally Lipschitz, we have

$$
\partial_{C} \phi(x)=\operatorname{co} \partial_{L} \phi(x) \text {. }
$$

As an exercise, we invite the reader to show that for $n=1$ and $\phi(x)=-|x|$, we have

$$
\partial_{P} \phi(0)=\emptyset, \partial_{L} \phi(0)=\{-1,+1\}, \partial_{C} \phi(0)=[-1,+1] .
$$

\section{Monotonicity along trajectories}

We now discuss the use of proximal calculus in connection with certain important monotonicity properties. We do this in the framework of a control system defined by a differential inclusion (19), together with a function $\phi$ which is lower semicontinuous and extended-valued.

We say that the pair $(\phi, F)$ is weakly decreasing if, for every $\alpha \in \operatorname{dom} \phi$, there exist a trajectory $x$ for $F$ and $\epsilon>0$ such that

$$
x(0)=\alpha, \phi(x(t)) \leq \phi(\alpha) \forall t \in[0, \epsilon]
$$

$(\phi, F)$ is said to be strongly increasing if, for any trajectory $x$, the function $t \mapsto \phi(x(t))$ is increasing, in the nonstrict sense of the word: $s<t \Longrightarrow \phi(x(s)) \leq \phi(x(t))$.

Similarly, $(\phi, F)$ is strongly decreasing if, for any trajectory $x$, the function $t \mapsto \phi(x(t))$ is decreasing (in the nonstrict sense).

The lower Hamiltonian $h$ corresponding to $F$ is defined as

$$
h(x, p):=\min \{\langle p, v\rangle: v \in F(x)\} .
$$

Note that this yields the same function as (4) when $F(x)=$ $f(x, U)$. The upper Hamiltonian $H$ for $F$ is defined by

$$
H(x, p):=\max \{\langle p, v\rangle: v \in F(x)\} .
$$

In the following results, we assume that $F$ has compact convex values, and is locally Lipschitz: for every bounded subset $S$, there exists $K=K(S)$ such that

$$
x, y \in S \Longrightarrow F(x) \subset F(y)+K|x-y| .
$$

The monotonicity properties introduced above are characterized by Hamilton-Jacobi inequalities in proximal terms: 
Theorem 8. $(\phi, F)$ is weakly decreasing if and only if $h(x, \zeta) \leq 0 \forall \zeta \in \partial_{P} \phi(x), \forall x \in \operatorname{dom} \phi$,

strongly increasing if and only if

$$
h(x, \zeta) \geq 0 \forall \zeta \in \partial_{P} \phi(x), \forall x \in \operatorname{dom} \phi,
$$

and strongly decreasing if and only if

$$
H(x, \zeta) \leq 0 \forall \zeta \in \partial_{P} \phi(x), \forall x \in \operatorname{dom} \phi .
$$

(The inequalities are understood to hold automatically at points $x$ for which $\partial_{P} \phi(x)$ is empty.)

\section{Invariance}

As a first illustration of the use of the theorem above, we deduce a useful characterization of sets having certain invariance properties relative to trajectories.

Let $S$ be a nonempty closed subset of $\mathbb{R}^{n}$. We say that the pair $(S, F)$ is weakly invariant if, for every $\alpha \in S$, there exists a trajectory $x$ for $F$ and $\epsilon>0$ such that

$$
x(0)=\alpha, x(t) \in S \quad \forall t \in[0, \epsilon] .
$$

$(S, F)$ is strongly invariant if, for any trajectory $x$,

$$
x(0) \in S \Longrightarrow x(t) \in S \quad \forall t \geq 0 .
$$

Theorem 9. $(S, F)$ is weakly invariant if and only if

$$
h(x, \zeta) \leq 0 \quad \forall \zeta \in N_{S}^{P}(x), \forall x \in S,
$$

and strongly invariant if and only if

$$
H(x, \zeta) \leq 0 \quad \forall \zeta \in N_{S}^{P}(x), \forall x \in S .
$$

Proof. This follows directly from Theorem 8 and from (24), upon noting that $(S, F)$ is weakly invariant if and only if $\left(I_{S}, F\right)$ is weakly decreasing, and similarly for strong invariance and strong decrease.

Remark: the property of weak invariance is sometimes called viability.

\section{Viscosity solutions}

In the next result, which illustrates the use of monotonicity in the theory of viscosity solutions, the multifunction $F$ is assumed to have linear growth.

Theorem 10. Let $\phi$ be a continuous positive-definite function which satisfies

$$
h(x, \zeta)=-1 \quad \forall \zeta \in \partial_{P} \phi(x), \forall x \neq 0,
$$

where $h$ is given by (25). Then $\phi$ is the minimal-time function $T(\cdot)$ for the system.

(Remark: The value of the minimal-time function $T$ is $+\infty$ at points which are not controllable to 0 in finite time. Note that in the above, it is not assumed a priori that the system is controllable, yet one obtains that $T(\cdot)$ is finitevalued.)

Proof. (Sketch) According to Theorem 8, or, more precisely, a certain domain-restricted version of it, the function

$$
(x, y) \mapsto \phi(x)+y
$$

is both strongly increasing and weakly decreasing on the domain $\mathbb{R}^{n} \backslash\{0\}$ for the augmented system $F(x) \times\{1\}$. Fix any $\alpha \neq 0$. For any trajectory $x$ joining $\alpha$ to 0 in time $\tau$, we have, by strong increase,

$$
\tau=\phi(x(\tau))+\tau \geq \phi(x(0))+0=\phi(\alpha) .
$$

It follows that $T(\alpha) \geq \phi(\alpha)$. By weak decrease, there exists a trajectory $x$ with $x(0)=\alpha$ such that

$$
\phi(x(t))+t \equiv \phi(\alpha) \quad \forall t \geq 0 .
$$

(This is where linear growth is used, to preclude blowup.) Then, at $t=\phi(\alpha)$, we must have $x=0$. This yields $T(\alpha) \leq \phi(\alpha)$, and completes the proof.

Example. We show that the minimal-time function $T$ proposed for the double integrator (8) satisfies the HamiltonJacobi equation of Theorem 10, which confirms that it is the minimal-time function of the system.

At points $(x, y) \neq 0$ not lying on the switching curve $S$, $T$ is locally $C^{2}$, so this can be done by calculating the derivative and substituting.

Suppose now that $(x, y)$ lies on the lower branch of $S$, and let $(p, q) \in \partial_{P} T(x, y)$. The proximal inequality then says that relative to nearby points to the right of $S$ (thus, for $\left.y^{2} \leq 2 x\right)$, the function $\left(x^{\prime}, y^{\prime}\right) \mapsto$

$y^{\prime}+\sqrt{2\left(y^{\prime}\right)^{2}+4 x^{\prime}}-p x^{\prime}-q y^{\prime}+\sigma\left\{\left|x^{\prime}-x\right|^{2}+\left|y^{\prime}-y\right|^{2}\right\}$

attains a minimum at $(x, y)$. Applying the classical Lagrange multiplier rule gives, for some $\lambda \geq 0$ :

$$
p=-4 \lambda-1 / y, q=4 \lambda y \leq 0 .
$$

It follows that

$$
h(x, y, p, q)=p y-|q|=-1,
$$

which is the desired conclusion. A similar argument applies on the upper branch of $S$.

\section{PROXIMAL CLF'S}

In Section 1 we recalled the concept of control-Lyapunov function in the classical smooth $\left(C^{1}\right)$ sense, and we also encountered an extension of the concept to merely continuous functions, by means of the Dini derivate. It turns out that for certain purposes (such as feedback design), we require an extension in proximal terms.

A control-Lyapunov function $V$ in the proximal sense (proximal clf) for the system $(*)$ refers to a continuous function $V: \mathbb{R}^{n} \rightarrow[0,+\infty)$ which is positive definite and proper and satisfies the following proximal version of infinitesimal decrease:

$$
\sup _{\zeta \in \partial_{P} V(x)} \min _{u \in U}\langle\zeta, f(x, u)\rangle<-W(x) \quad \forall x \neq 0
$$

for some rate function $W$ (as defined earlier). This inequality holds automatically when $\partial_{P} V(x)$ is empty (by convention, and in accord with the usual interpretation of the supremum over the empty set as $-\infty$.)

A $C^{1}$ clf is necessarily a proximal clf, since, when $\nabla V(x)$ exists, we have $\partial_{P} V(x) \subset\{\nabla V(x)\}$. As regards the relationship with Dini clf's, we have: 
Proposition 11. A Dini clf is also a proximal clf. If the system is relaxed, the converse holds as well.

Proof. (Sketch) Let $V$ be a Dini clf, and let $\zeta \in \partial_{P} V(x)$ Then, directly from the definition of proximal subgradient, we derive

$$
d V(x ; v) \geq\langle\zeta, v\rangle \forall v \in \mathbb{R}^{n} .
$$

It follows from this that $V$ is a proximal clf. As for the converse, see (Clarke et al., 1998, p. 210).

As a corollary of the proof, we note the following hybrid criterion that is useful in confirming that a given function is a proximal clf:

Corollary 12. Let $V$ be continuous, proper, and positive definite, and suppose that for some rate function $W$ we have, at each point $x \neq 0$, either

$$
\sup _{\zeta \in \partial_{P} V(x)} \min _{u \in U}\langle\zeta, f(x, u)\rangle<-W(x)
$$

(which is the case if $\partial_{P} V(x)$ is empty), or

$$
\min _{u \in U} d V(x ; f(x, u))<-W(x) .
$$

Then $V$ is a control-Lyapunov function in the proximal sense.

The following result implies that the existence of a clf in any of the three senses we have seen (smooth, Dini, or proximal) implies the asymptotic controllability of the system, since the proximal sense is the weakest of the three.

Theorem 13. If the system admits a control-Lyapunov function in the proximal sense, then it is asymptotically controllable.

Proof. (Sketch) It suffices to prove the theorem when the system is relaxed. This is done by observing that (26) is precisely the Hamiltonian condition characterizing the weak decrease property (see Theorem 8 ) for the function $(x, y) \mapsto V(x)+y$ relative to the augmented system $x^{\prime}=f(x, u), y^{\prime}=W(x)$ (where the rate function $W$ has been chosen to be suitably regular). It follows that from any initial point $\alpha$ there is a trajectory $x$ beginning at $\alpha$ such that

$$
V(x(t))+\int_{0}^{t} W(x(\tau)) d \tau \leq V(\alpha) \quad \forall t \geq 0 .
$$

One then shows that this implies $x(t) \rightarrow 0$, whence $V(x(t)) \rightarrow 0$ by continuity. Asymptotic controllability follows from this; see (Clarke et al., 1998, pp. 213-214) for details.

Example. We show that the following function $V_{1}$ is a proximal clf for the nonholonomic integrator (14):

$$
\begin{aligned}
V_{1}\left(x_{1}, x_{2}, x_{3}\right) & :=\left\{\sqrt{x_{1}^{2}+x_{2}^{2}}-\left|x_{3}\right|\right\}^{2}+x_{3}^{2} \\
& =x_{1}^{2}+x_{2}^{2}+2 x_{3}^{2}-2\left|x_{3}\right| \sqrt{x_{1}^{2}+x_{2}^{2}} .
\end{aligned}
$$

Proof. We omit the proof that $V_{1}$ is positive definite and proper. We set

$$
\sigma:=\sqrt{x_{1}^{2}+x_{2}^{2}}
$$

for notational convenience.

We consider first the points $x$ for which $\sigma \neq 0$ and $x_{3} \neq 0$. At such points, we have $\partial_{P} V_{1}(x)=\nabla V_{1}(x)$, since $V_{1}$ is locally $C^{2}$. It follows that verifying infinitesimal decrease at such points in either the proximal, Dini, or smooth sense amounts to the same thing. We calculate $\nabla V_{1}(x)$ :

$$
\left[2 x_{1}-2\left|x_{3}\right| x_{1} / \sigma, 2 x_{2}-2\left|x_{3}\right| x_{2} / \sigma, 4 x_{3}-2 \sigma \operatorname{sgn}\left(x_{3}\right)\right],
$$

where $\operatorname{sgn}(y):=y /|y|$ is the usual signum function. Let $u_{1}=x_{1} / \sigma$ and $u_{2}=x_{2} / \sigma$. Then the admissible control values $\pm\left(u_{1}, u_{2}\right) \in U$ give rise to an inner product $\left\langle\nabla V_{1}(x), f(x, u)\right\rangle$ equal to $\pm 2\left(\sigma-\left|x_{3}\right|\right)$.

Now take $v_{1}=x_{2} / \sigma$ and $v_{2}=-x_{1} / \sigma$. Then the admissible control values $\pm\left(v_{1}, v_{2}\right) \in U$ give rise to corresponding inner products $\pm 2 \sigma\left(\sigma \operatorname{sgn}\left(x_{3}\right)-2 x_{3}\right)$. It follows that

$$
\min _{u \in U}\left\langle\nabla V_{1}(x), f(x, u)\right\rangle \leq-W(x),
$$

where $W(x)$ is defined to be

$$
2 \max \left\{|\sigma-| x_{3}||, \sigma\left|\sigma \operatorname{sgn}\left(x_{3}\right)-2 x_{3}\right|\right\} .
$$

The required conclusion now follows from the fact that $W$ is a rate function: $W$ is positive and bounded away from 0 on any compact subset of $\mathbb{R}^{n} \backslash\{0\}$.

There remain the other (nonzero) points to consider. If $x_{3}=0$, then $\sigma \neq 0$, and if $\sigma=0$, then $x_{3} \neq 0$. In either case, we see directly from the last term in (27) that $\partial_{P} V_{1}(x)=\emptyset$, so that the first criterion in Corollary 12 is satisfied, which concludes the proof.

\section{REGULARITY OF CLF'S}

As we have seen, asymptotic controllability cannot be characterized by smooth clf's. The alternative so far has been to introduce Dini and proximal clf's, which are merely continuous. This raises a natural question: given an asymptotically controllable system, what is the greatest degree of regularity which is always attainable by some clf for the system? Clearly, the answer lies somewhere between continuous and $C^{1}$, while being strictly less than the latter.

A longstanding conjecture in the subject has been that a locally Lipschitz clf $V$ is always available. This was proved in 2000 by L. Rifford, who went on to prove more: there is always a clf which is semiconcave on $\mathbb{R}^{n} \backslash\{0\}$. This is a useful and much-studied property in pde's and optimal control (see Cannarsa and Sinestrari (2004)).

We give in this section a relatively brief and self-contained treatment of the topic based on the generalized gradient, and tuned to our specific purposes. The utility of knowing that a clf is locally Lipschitz or semiconcave will be clarified later in connection with feedback design.

Definition. Let $\phi: \mathbb{R}^{n} \rightarrow \mathbb{R}$ be a function and $x \in \mathbb{R}^{n}$ a given point. We say that $\phi$ has property SC at $x$ provided that there exist positive constants $r, L, \sigma, \eta$ such that $\phi$ is Lipschitz of rank $L$ on $B(x, r)$ :

$$
|\phi(y)-\phi(z)| \leq L|y-z| \forall y, z \in B(x, r),
$$

and such that, for almost all $y, z \in B^{\circ}(x, r)$, we have

$$
\phi(y)-\phi(z)-\langle\nabla \phi(z), y-z\rangle \leq \sigma|y-z|^{1+\eta} .
$$


When (SC1) holds, then $\phi$ is differentiable almost everywhere in $B(x, r)$ by Rademacher's Theorem, so that the condition (SC2) makes sense. If it is desired to take note of the parameters involved, then we say that $\phi$ has property $\mathrm{SC}$ with constants $(r, L, \sigma, \eta)$.

It turns out that the generalized gradient $\partial_{C} \phi$ of Section 3 is useful in characterizing such functions, in analyzing their properties, and in establishing how SC is preserved by such operations as sums, compositions and products. Recall that a function $h$ is said to be Hölder continuous on a set $S$ if there exist $K, \alpha>0$ such that

$$
|h(x)-h(y)| \leq K|x-y|^{\alpha} \forall x, y \in S .
$$

We list some basic facts regarding the property SC. Theorem 14 .

1. $\phi$ has property $\mathrm{SC}$ at $x$ with constants $(r, L, \sigma, \eta)$ iff (SC1) holds together with

$$
\begin{aligned}
& \phi(y)-\phi(z)-\langle\zeta, y-z\rangle \leq \sigma|y-z|^{1+\eta} \\
& \forall y, z \in B^{\circ}(x, r), \zeta \in \partial_{C} \phi(z) .
\end{aligned}
$$

2. If $\phi$ satisfies SC at each point $x$ of an open set $\Omega$, with constants that may depend on $x$, then for any compact subset $X$ of $\Omega$ there exist positive constants $L, \sigma, \eta$ and $\rho$ such that $\phi$ is Lipschitz of rank $L$ on $X$ and

$$
\begin{aligned}
y, z \in X, & |y-z|<\rho, \zeta \in \partial_{C} \phi(z) \\
& \Longrightarrow \phi(y)-\phi(z)-\langle\zeta, y-z\rangle \leq \sigma|y-z|^{1+\eta} .
\end{aligned}
$$

3. If $\phi$ is differentiable near $x$ and $\nabla \phi$ is Hölder continuous near $x$, then $\phi$ satisfies SC at $x$. This holds in particular when $\phi$ is $C^{2}$ near $x$.

4. If $\phi$ coincides near $x$ with a function which is concave, then $\phi$ satisfies SC at $x$, with $\sigma=0$.

5 . The positive linear combination (and in particular, the sum) of a finite number of functions each of which satisfies $\mathrm{SC}$ at $x$ also satisfies $\mathrm{SC}$ at $x$.

6. If $\phi=g \circ h$, where $h: \mathbb{R}^{n} \rightarrow \mathbb{R}^{m}$ is differentiable near $x$ and $D h$ is Hölder continuous near $x$, and where $g: \mathbb{R}^{m} \rightarrow \mathbb{R}$ is concave, then $\phi$ satisfies SC at $x$.

7. If $\phi=g \circ h$, where $h: \mathbb{R}^{n} \rightarrow \mathbb{R}$ is concave, and where $g: \mathbb{R} \rightarrow \mathbb{R}$ is differentiable near $h(x)$ and $D g$ is Hölder continuous near $h(x)$, then $\phi$ satisfies SC at $x$.

8. If $\phi=g h$, where $h$ coincides near $x$ with a function which is convex, and where $g: \mathbb{R}^{n} \rightarrow(-\infty, 0]$ is differentiable near $x$ and $D g$ is Hölder continuous near $x$, then $\phi$ satisfies $\mathrm{SC}$ at $x$.

9. If $\phi=g h$, where $g$ is differentiable near $x$ and $D g$ is Hölder continuous near $x$, with $g(x)>0$, and where $h$ is concave, then $\phi$ satisfies $\mathrm{SC}$ at $x$.

10. If $\phi=\min \phi_{i}$, where $\left\{\phi_{i}\right\}$ is a finite family of functions each of which satisfies $(*)$ at $x$, then $\phi$ satisfies SC at $x$.

11. If $\phi=\inf _{\alpha \in A} \phi_{\alpha}$ is finite ( $A$ countable), where each $\phi_{\alpha}$ satisfies $\mathrm{SC}$ at $x$ with the same constants $(r, L, \sigma, \eta)$, then $\phi$ also satisfies SC at $x$ with those constants.

12. If $\phi$ satisfies SC at $x$ with constants $(r, L, \sigma, 2)$, then there is a concave function $g: B(x, r) \rightarrow \mathbb{R}$ with Lipschitz constant $K_{g}:=L+2 \sigma r$ such that $\phi(y)=g(y)+\sigma|y-x|^{2}$ for $y \in B(x, r)$. Conversely, if $\phi$ admits such a representation, where $g$ has Lipschitz constant $K_{g}$, then $\phi$ satisfies SC at $x$ with constants $(r, L, \sigma, 2)$, where $L:=K_{g}+2 \sigma r$.

13. If $\phi$ satisfies SC at $x$, then the directional derivative $\phi^{\prime}(x ; v)$ exists for each $v$, and one has

$$
d \phi(x ; v)=\phi^{\prime}(x ; v)=\min _{\zeta \in \partial_{C} \phi(x)}\langle\zeta, v\rangle \quad \forall v \in \mathbb{R}^{n} .
$$

We omit the proof, which is largely routine. We comment, however, that Part (11) follows from (Clarke, 1983, Theorem 2.8.6), and that the function $g$ in Part (12) is given by

$$
g(y):=\min \phi(z)+\langle\zeta, y-z\rangle+\sigma\langle z-x, z+x-2 y\rangle,
$$

where the minimum is taken over $z \in B^{\circ}(x, r), \zeta \in \partial_{C} \phi(z)$. The penultimate item above shows that property $\mathrm{SC}$ is at heart a type of semiconcavity, and explains (perhaps) the origin of this terminology. We shall say for simplicity that a clf is semiconcave if it satisfies property SC on $\mathbb{R}^{n} \backslash\{0\}$.

In the presence of property SC, Dini and proximal clf's coincide:

Proposition 15. If $V$ satisfies property SC on $\mathbb{R}^{n} \backslash\{0\}$, then $V$ is a proximal clf if and only if it is a Dini clf.

Proof. (Sketch) We already know that a Dini clf is a proximal clf, by Prop. 11. Let $V$ be a proximal clf. Then, for every $x \neq 0$, we have (by an evident limiting argument)

$$
\max _{\zeta \in \partial_{L} V(x)} \min _{u \in U}\langle\zeta, f(x, u)\rangle \leq-W(x) .
$$

Then, by Part (13) of Theorem 14, we have

$$
\begin{aligned}
\min _{u \in U} d V(x ; f(x, u))=\min _{u \in U} \min _{\zeta \in \partial_{C} V(x)}\langle\zeta, f(x, u)\rangle \\
=\min _{u \in U} \min _{\zeta \in \partial_{L} V(x)}\langle\zeta, f(x, u)\rangle \\
=\min _{\zeta \in \partial_{L} V(x)} \min _{u \in U}\langle\zeta, f(x, u)\rangle \leq-W(x),
\end{aligned}
$$

which confirms that $V$ is a Dini clf.

Example. We showed above that the function $V_{1}$ of (27) is a proximal clf for the nonholonomic integrator (14). We now show that it is semiconcave (that is, that it satisfies property $\mathrm{SC}$ at nonzero points).

Proof. At a point $x$ where $\left(x_{1}, x_{2}\right) \neq 0$, the final term in the expression (27) satisfies property SC by Part (9) of Theorem 14. At a point $x$ where $\left(x_{1}, x_{2}\right)=0, x_{3} \neq 0$, the same term satisfies property SC by Part (8) of the theorem. Since all the other terms in (27) are $C^{2}$, it now follows from Parts (3) and (5) of the theorem that $V_{1}$ has property $\mathrm{SC}$ at all nonzero points.

Example. Another locally Lipschitz Lyapunov function in the Dini sense for the nonholonomic integrator (14) is the following:

$$
V_{2}(x):=\max \left\{\sqrt{x_{1}^{2}+x_{2}^{2}},\left|x_{3}\right|-\sqrt{x_{1}^{2}+x_{2}^{2}}\right\} .
$$

(This can be shown by the same proof technique used for $V_{1}$.) We claim that $V_{2}$ fails to satisfy $\mathrm{SC}$ at certain points. 
Proof. At the point $x:=(1,0,2), \partial_{C} V_{2}(x)$ is given by the segment with endpoints $(1,0,0)$ and $(-1,0,1)$, by Part (2) of Theorem 6. If SC holds at $x$, then, by Part (1) of Theorem 14, taking $z:=x$ and $\zeta=(-1,0,1)$, we would have locally

$$
\sqrt{y_{1}^{2}+y_{2}^{2}} \leq V_{2}(y) \leq V_{2}(x)+\langle\zeta, y-x\rangle+\sigma|y-x|^{1+\eta} .
$$

Taking $y=(1,0,2-\epsilon)$ for all suitably small $\epsilon>0$, this reduces to $1 \leq \sigma \epsilon^{\eta}$, which is absurd.

In Section 8 we explain why the facts proven above imply that $V_{1}$ is a 'better' clf than $V_{2}$.

\section{CONVERSE THEOREMS}

A 'converse theorem' is a result stating that an asymptotically controllable system must admit a clf (of some type). As (Sontag, 1990, p. 259) puts it:

"In any case, all converse Lyapunov functions are purely existential, and are of no use in guiding the search for a Lyapunov function. The search for such functions is more of an art than a science, and good physical insight into a given system plus a good amount of trial and error is typically the only way to proceed. There are, however, many heuristics that help...another possibility is to build control-Lyapunov functions recursively, via 'backstepping'..."

It turns out, however, that the techniques by which converse Lyapunov theorems have been proved, which tend to lead to nonsmooth functions, do offer some guidance when we work in a nonsmooth setting. We shall describe two such approaches.

\section{The value function technique}

Suppose that system $(*)$ is asymptotically controllable. Fix $r>0$, and, for a given rate function $W$, define

$$
\phi(\alpha):=\min \int_{0}^{T} W(x(t)) d t,
$$

where the minimum is taken over all trajectories $x$ of the system satisfying $x(0)=\alpha$ and $x(T) \in B(0, r)$, and where $T$ is free. (Thus, when $W$ is identically 1 , the function $\phi$ is the minimal-time function for the target $B(0, r)$.) Note that the asymptotic controllability guarantees that there are admissible trajectories for the optimal control problem in question. Under certain familiar hypotheses (notably that the system is relaxed), an optimal trajectory then exists.

The function $\phi$ is an example of a value function: at $\alpha$, its value coincides with the value (that is, the minimum) of a certain optimization problem in which $\alpha$ is a parameter. Such functions play a central role in pde's, optimization, and differential games. We claim that $\phi$ is rather close to being a control-Lyapunov function for the system. But in which sense? Certainly not the $C^{1}$ sense, for value functions are notoriously nonsmooth.

Let us show that $\phi$ is (almost) a proximal clf. Under mild conditions on $W$, we can arrange for $\phi$ to be proper as well as positive definite relative to the ball $B(0, r)$ (rather than the origin). We claim that the function

$$
(x, y) \mapsto V(x)+y
$$

is weakly decreasing (see Section 3) relative to the augmented system $x^{\prime}=f(x, u), y^{\prime}=W(x)$. To see this (directly from the definition), let $(\alpha, \beta)$ be a given initial condition for the augmented state. Let $x$ be a trajectory attaining the minimum in the definition of $\phi(\alpha)$. Then, by the reasoning known as the Principle of Optimality, we have

$$
\phi(x(t))=\int_{t}^{T} W(x(\tau)) d \tau, \quad \forall t \in[0, T] .
$$

If we define

$$
y(t):=\beta+\int_{0}^{t} W(x(\tau)) d \tau,
$$

then $(x, y)$ is an augmented trajectory satisfying

$$
(x(0), y(0))=(\alpha, \beta),
$$

and the preceding equality implies

$$
\phi(x(t))+y(t)=\phi(\alpha)+\beta, \quad \forall t \in[0, T],
$$

which confirms weak decrease, as claimed. Observing that

$$
\partial_{P}(V(x)+y)=\partial_{P} V(x) \times\{1\},
$$

we deduce from Theorem 8 that

$$
\sup _{\zeta \in \partial_{P} V(x)} \min _{u \in U}\langle\zeta, f(x, u)\rangle+W(x) \leq 0 \quad \forall x \notin B(0, r) .
$$

If $W$ has been chosen to be a rate function (relative to the target $B(0, r)$ ), we obtain strict inequality by replacing $W$ by $W / 2$ (say).

It follows that $\phi$ is a proximal clf for the system, when the equilibrium 0 is replaced by the set $B(0, r)$.

Replacing $B(0, r)$ by $\{0\}$, so as to get a precise converse theorem, requires more work. One route is described in Sontag (1983), and another proceeds as follows: In Clarke et al. (2000) it was shown that certain value functions along the lines of the ones described above give rise to practical Lyapunov functions (that is, assuring controllability to arbitrary small balls $B(0, r)$ ); the system was modified in order to make these value functions locally Lipschitz. Building upon this, L. Rifford was able to combine a countable family of such functions in order to construct a true locally Lipschitz clf for the origin (in the proximal sense), and even a semiconcave one.

We remark that value functions such as $\phi$ underlie the philosophy of the model-predictive (or receding horizon) methods for numerical stabilization (see for example Fontes and Magni (2003)). The possible semiconcavity of such functions has been extensively studied (because of its importance in viscosity solutions; see Cannarsa and Sinestrari (2004)). One hypothesis required in these results is that the target set satisfy the 'inner ball condition'. This holds when the target is $B(0, r)$, but not when the target is a single point. The need for this hypothesis is illustrated by the minimal-time function $T(\cdot)$ associated with the double integrator (8), which is not semiconcave (as evidenced 
by the fact that it fails to be locally Lipschitz along the switching curve).

\section{Practical semiglobal controllability}

The discussion above indicates why it is technically simpler to find clf's for a neighborhood of 0 rather than to 0 itself. In a similar vein, it will simplify matters considerably later on to seek feedbacks that achieve 'practical semiglobal' stabilization (from a given ball $B(0, R)$ to a prescribed ball $B(0, r)$ ) rather than asymptotically to the origin from any initial condition. In reality, the stabilization will take place not between balls, but between level sets of a given clf. For the time being, let us merely note the following practical semiglobal version of Theorem 13:

Theorem 16. Let $V$ be a continuous function such that the level set $\{x: V(x) \leq E\}$ is compact, and suppose that for some $e<E$ and $\omega>0$, there is a neighborhood $\Omega$ of the set

such that

$$
\{x: e \leq V(x) \leq E\}
$$

$$
x \in \Omega, \zeta \in \partial_{P} V(x) \Longrightarrow \min _{u \in U}\langle\zeta, f(x, u)\rangle \leq-\omega .
$$

Then, for any $\alpha \in\{x: V(x) \leq E\}$, and for any $\epsilon>0$, there is a trajectory $x$ having $x(0)=\alpha$ such that, for some $T$ satisfying

$$
0 \leq T \leq(V(\alpha)-e) / \omega \leq(E-e) / \omega,
$$

we have $V(x(T)) \leq e+\epsilon$.

Proof. (Sketch) Because relaxed trajectories are uniformly approximated by original ones with the same initial value, it suffices to prove the theorem for relaxed trajectories, with $\epsilon=0$. Theorem 8 (or its domain-restricted version) implies the existence of a relaxed trajectory $x$ beginning at $\alpha$ for which

$$
V(x(t))+\omega t \leq V(\alpha) \quad \forall t \geq 0,
$$

up to the first time $T$ for which $V(x(t)) \leq e$ (blow-up is precluded by the boundedness of the level set $\{V \leq E\}$ ). This yields the desired conclusion.

\section{The field of trajectories technique}

The minimal-time function is constructed by following time-optimal trajectories. However, it is possible to construct Lyapunov functions by other than time-optimal ones, as we now describe in an apparently new formalism.

Let $\mathcal{T}$ be a given target: a nonempty closed subset of $\mathbb{R}^{n}$. We say that a family $\left\{x_{\alpha}\right\}$ is a field of trajectories (for $\mathcal{T}$ ) if:

- For every $\alpha \in \mathbb{R}^{n} \backslash \mathcal{T}, x_{\alpha}$ is a trajectory on an interval $\left[0, T_{\alpha}\right]$ with $x_{\alpha}(0)=\alpha$, and $T_{\alpha} \in(0,+\infty)$ is the first time $t$ for which $x_{\alpha}(t) \in \mathcal{T}$;

- For some $\delta_{\alpha}>0, x_{\alpha}$ is differentiable on $\left(0, \delta_{\alpha}\right)$;

- The family is consistent: for $s>0$, the trajectory $x(\cdot)$ associated to the point $x_{\alpha}(s)$ is $x(t)=x_{\alpha}(t+s), t \geq 0$;
- The function $\alpha \mapsto T_{\alpha}$ is continuous on $R^{n} \backslash \mathcal{T}$, and $T_{\alpha} \rightarrow 0$ as $\alpha \rightarrow \mathcal{T}$.

This is not a field in the classical sense of the calculus of variations, since we do not impose one-to-one covering of a region by the trajectories. However, it follows that $V(\alpha):=T_{\alpha}$ has the essential features of a clf (relative to the target $\mathcal{T}$ ), when we define $V$ to be 0 on the set $\mathcal{T}$ itself. Evidently, $V$ is then continuous and positive definite relative to $\mathcal{T}$. The following confirms infinitesimal decrease in the Dini sense on $\mathbb{R}^{n} \backslash \mathcal{T}$ :

Proposition 17. For every $x \in \mathbb{R}^{n} \backslash \mathcal{T}$, we have

$$
\min _{u \in U} d V(x ; f(x, u)) \leq-1 .
$$

Proof. Fix any $\alpha \notin \mathcal{T}$. Let $t_{i}$ be a positive sequence decreasing to 0 . It follows from the mean value theorem (and the basic hypotheses) that, for some subsequence (we do not relabel), for some $u \in U$, the points

$$
w_{i}:=\left(x_{\alpha}\left(t_{i}\right)-x_{\alpha}(0)\right) / t_{i}
$$

converge to $f(\alpha, u)$. We observe that (for all $i$ sufficiently large)

$$
\begin{aligned}
\left(V\left(\alpha+t_{i} w_{i}\right)-V(\alpha)\right) / t_{i} & =\left(V\left(x_{\alpha}\left(t_{i}\right)\right)-V(\alpha)\right) / t_{i} \\
& =\left(T_{\alpha}-t_{i}-T_{\alpha}\right) / t_{i}=-1 .
\end{aligned}
$$

The result follows.

Of course the very fact that we may exhibit such a field confirms in itself that we have controllability to $\mathcal{T}$. It may seem that a clf is hardly necessary after that. But confirming controllability is not our sole aim: Lyapunov functions play a role in designing stabilizing feedback. We shall see later how to use $V$ in order to do so.

Example. We consider the following system:

$$
\begin{aligned}
x^{\prime} & =u y, y^{\prime}=v \\
(u, v) & \in U:=\{(0,1),(0,-1),(1,0),(-1,0)\},
\end{aligned}
$$

in which $n=2, m=2$. We are interested in controllability to the unit ball $B(0,1)$.

We proceed to find a clf for the system by the field of trajectories method, relative to the target

$$
\mathcal{T}:=\{(0,1),(0,-1)\} \subset B(0,1) .
$$

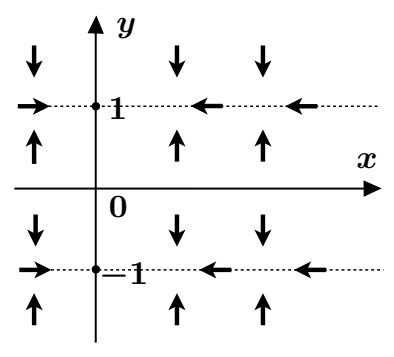

Fig. 6. A vector field
We define the following strategy for reaching $\mathcal{T}$ in finite time from an initial state $(\alpha, \beta) \notin \mathcal{T}$ (see Fig. 6): First, take $(u, v)= \pm(0,1)$ until $|y|=1$ is attained; time required: ||$\beta|-1|$. Then take $(u, v)= \pm(1,0)$ until $x=0$; time required: $|\alpha|$.

The resulting function $V$ is given explicitly by

$$
V(\alpha, \beta)=|| \beta|-1|+|\alpha| .
$$

This defines a Dini clf relative to $\mathcal{T}$. (In more complex examples $V$ may be implicitly defined.) We return to this example later. 


\section{Finite-time controllability}

So far we have been concerned with possibly asymptotic approach to the origin. There is interest in being able to assert that the origin can be reached in finite time. If such is the case from any initial condition, then we say that the system is controllable in finite time (to 0).

There is a well-studied local version of this property that bears the name small-time local controllability (STLC for short). A number of verifiable criteria exist which imply that the system has property STLC, which is stronger than local Lyapunov stability; see Bardi and Capuzzo-Dolcetta (1997).

Theorem 18. Let the system $(*)$ be relaxed and have linear growth, as well as property STLC. Then it is controllable in finite time if and only if there exists a proximal controlLyapunov function $V$ having rate function $W \equiv 1$.

Proof. (Sketch) The proof of the theorem is centered upon the minimal time function $T(\cdot)$, which is known to be continuous when STLC holds. If the system is controllable in finite time, then $T$ is the required clf: weak infinitesimal decrease follows from the fact that $T$ satisfies the proximal Hamilton-Jacobi equation. The sufficiency follows much as in the proof of Theorem 13: we deduce the existence of a trajectory $x$ for which $V(x(t))+t$ is nonincreasing as long as $x(t) \neq 0$; this implies that $x(\tau)$ equals 0 for some $\tau \leq V(x(0))$.

\section{DISCONTINUOUS STABILIZERS}

We have seen earlier that even when the system $(*)$ is asymptotically controllable, it may fail to admit a continuous feedback $k$ which stabilizes it. It is natural therefore to consider discontinuous feedbacks. This raises the issue of giving a meaning to the differential equation $x^{\prime}=f(x, k(x))$ when the right side is not a continuous function of $x$.

The Filippov solution concept for differential equations $x^{\prime}=g(x)$, where $g$ is discontinuous, was reviewed in Section 2. As explained there, Filippov solutions can fail to reflect the dynamics by generating superfluous trajectories that are not meaningful. The consequence of this phenomenon for the stabilization issue is that Filippov solutions are inappropriate as a means of interpreting $x^{\prime}=f(x, k(x))$ when $k$ is discontinuous.

As evidence of this, we remark that it has been shown that Theorem 4 continues to hold for discontinuous feedbacks when they are interpreted in the Filippov sense (Ryan (1994); Coron and Rosier (1994)). Thus the nonholonomic integrator, for example, fails to admit a feedback that stabilizes the Filippov solutions.

The sample-and-hold implementation of a (possibly discontinuous) feedback was also described in Section 2. Because a $\pi$-trajectory $x$ generated from the sample-and-hold procedure is an actual state trajectory corresponding to a piecewise constant open-loop control, it is a physically meaningful one.

Definition. We say that a feedback $k(x)$ (continuous or not) stabilizes the system in the sample-and-hold sense provided that, for every ball $B(0, R)$ of initial values to be stabilized, and for every target ball $B(0, r)$ to be aimed for, there exist positive numbers $\delta$ and $T$ depending only upon $r$ and $R$, and a number $C$ depending only on $R$, such that, for any initial value $x_{0} \in B(0, R)$, for any partition $\pi$ of diameter less than $\delta$, the corresponding $\pi$-trajectory $x$ beginning at $x_{0}$ satisfies

$$
|x(t)| \leq C \quad \forall t \geq 0, x(t) \in B(0, r) \quad \forall t \geq T .
$$

Note that the same feedback $k$ is involved here, whatever $r$ and $R$ are chosen. However, in order to attain the desired stabilization, the partition diameter $\delta$ will have to shrink as $R$ increases and as $r$ decreases. This simply reflects the need to sample the state at a higher rate in such cases, a natural and inevitable consideration.

If $k$ is a continuous feedback that stabilizes the system in the classical sense (that is, by making $x^{\prime}=f(x, k(x))$ asymptotically stable), then it also stabilizes the system in the sample-and-hold sense, so we are dealing with a faithful generalization of the classical notion.

The question of the equivalence between asymptotic controllability and feedback stabilizability was settled in 1997 by Clarke, Ledyaev, Sontag, and Subbotin, in terms of sample-and-hold:

Theorem 19. (Clarke et al. (1997)). The system is asymptotically controllable if and only if there exists a (possibly discontinuous) feedback $k$ which stabilizes it in the sampleand-hold sense.

The proof of the theorem uses proximal aiming, a method which is difficult to implement in practice. One of our principal goals here is to show how stabilizing feedbacks can be defined in a simpler way directly from a clf.

The use of possibly discontinuous feedback has arisen in other control contexts besides the one considered here. In linear time-optimal control, one can find discontinuous feedback syntheses as far back as the classical book of Pontryagin et al. (1962), as we have seen with the double integrator. In these cases, the feedback is invariably piecewise constant relative to certain partitions of state space, and solutions either follow the switching surfaces or cross them transversally, so the issue of defining the solution in other than a classical sense does not arise.

Somewhat related to this is the approach that defines a multivalued feedback law (see for example Berkovitz (1989)). In stochastic control, discontinuous feedbacks are the norm, with the solution understood in terms of stochastic differential equations. In a similar vein, in the control of certain linear partial differential equations, discontinuous feedbacks can be interpreted in a distributional sense. These cases are all unrelated to the one under discussion.

In contrast, the sample-and-hold implementation of discontinuous strategies in differential games is well-known: see Krasovskii and Subbotin (1988). We remark that in games of pusuit and evasion (for example), it is known that, in general, it is not possible to achieve the result of a discontinuous optimal strategy to within any tolerance by means of a continuous stategy (thus there can be a positive 
unbridgeable gap between the performance of continuous and discontinuous feedbacks).

\section{The thin set fallacy}

The classical solution concept for the differential equation $x^{\prime}=g(x)$, where $g$ is continuous, automatically incorporates a robustness to implementation and error. As an illustration of this, recall that if we proceed to calculate a solution using Euler's method, for example, then, for sufficiently fine partitions, we arrive at an answer which approximates the exact solution to any specified tolerance. This property, as well as other familiar ones such as continuous dependence on the initial condition, does not necessarily carry over to the case in which $g$ is discontinuous.

The consequence of this as regards system $(*)$ is that care must be taken that the stabilizing feedbacks we define are meaningful. This is a new type of consideration that we proceed to illustrate.

Example. Let us consider a control system in the plane having the property that the set of available velocities always allows one to move along circles centered at the origin, in either direction, at a constant rate. (We do not define the system explicitly.) Suppose furthermore that, for points on the positive $x_{2}$-axis, movement along that axis toward the origin is possible, at a constant rate.

We now consider the following feedback strategy as a means of driving the state to the origin (see Fig. 7). From any point $(\alpha, \beta) \neq 0$ in the plane, follow the circle centered at 0 that is determined by $(\alpha, \beta)$, until the positive $x_{2}$ axis is reached, using clockwise movement if $\alpha<0$, and counterclockwise if $\alpha>0$. Then descend the $x_{2}$-axis to the origin. This field of trajectories (see Section 6) implicitly defines the corresponding (discontinuous) feedback $k$.

The feedback appears to be effective as regards the differential equation: $k$ is presumably piecewise smooth (with at most one switch) along the resulting trajectories; these are well-defined in the classical sense, and arrive at the origin in finite time.

Note that, on the face of it, the feedback does not seem very different in nature from the minimal-time synthesis for the double integrator (see Fig. 1).

Yet to accept it as a stabilizing feedback would be absurd, we would argue. The problem is that the effect of the feedback depends upon the state being exactly on the $x_{2}$-axis at some point. This will essentially never happen in any numerical implementation (since the axis has measure $0)$. The result of the slightest overshoot is that the state trajectory, once it is

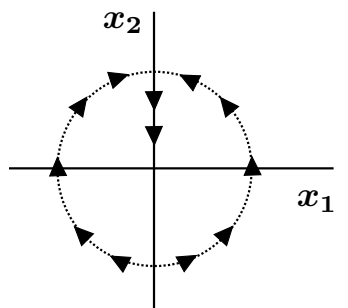

Fig. 7. A dubious stabilization strategy near the $x_{2}$-axis, will dither around it, rather than converge to the origin.
This illustrates the importance of avoiding what logicians refer to as the 'fallacy of misplaced concreteness': we must bear in mind that the symbolism $x^{\prime}=f(x, k(x))$ actually refers to an implementation procedure for using $k$. When $k$ is continuous, this can be (and usually is) ignored, for the reasons we have explained.

One of the principal virtues of the sample-and-hold approach is that it forces us to consider implementation, by its very definition. We remark that the feedback in the example does not stabilize the system in the sample-andhold sense. Thus, the natural feedback associated to a field of trajectories is not necessarily a good one. Nonetheless, as we shall see, the clf that it generates can be used in a certain way to define a meaningful feedback.

The danger we have identified here, one to which the literature is, alas, not immune, could be termed the thin set fallacy: using a feedback whose global effect depends on its values on some set of measure zero.

We summarize for now by saying that, in contrast to the case of continuous feedbacks, some discontinuous feedbacks will have robustness to error and implementation, and others will not. The issue needs to be studied separately, and the sample-and-hold approach leads to a rigorous analysis. We shall demonstrate this later in the case of sliding-mode control, which is indeed based upon using a discontinuous feedback to drive the state to a thin set.

\section{FEEDBACK DESIGN}

We base the design of stabilizing feedback upon a given clf for the system. The specific method will vary according to the regularity of the clf that we possess: smooth, semiconcave, locally Lipschitz, or merely continuous. We focus attention on practical semiglobal stabilization.

\section{Smooth clf's}

The case in which there exists a smooth clf is an important one. Although, as we have seen, there may not exist a continuous stabilizer even then, the steepest descent design (see below) is appealing, and the proof that sample-andhold stabilization takes place is a template for other, less evident cases.

Let $V$ be a clf for the system $(*)$, where $V$ is $C^{1}$ on the set $\mathbb{R}^{n} \backslash\{0\}$. Thus $V$ is continuous, proper, and positive definite, and there exists a rate function $W$ for $V$ such that the infinitesimal decrease condition holds:

$$
\inf _{u \in U}\langle\nabla V(x), f(x, u)\rangle<-W(x) \quad x \neq 0 .
$$

We recall that a rate function refers to a positive function $W$ on $\mathbb{R}^{n} \backslash\{0\}$ which is bounded away from 0 on compact subsets of $\mathbb{R}^{n} \backslash\{0\}$.

Notation: $\operatorname{argmin}_{v \in V} g(v)$ refers to the set of points in $V$ at which $g$ attains a minimum.

A natural way to define a feedback $k$ is by steepest descent: for each $x \neq 0$, choose

$$
k(x) \in \operatorname{argmin}_{u \in U}\langle\nabla V(x), f(x, u)\rangle .
$$

It follows then that 


$$
\langle\nabla V(x), f(x, k(x))\rangle<-W(x) \quad \forall x \neq 0 .
$$

In what follows, it is actually this inequality that is used. By extension, even if $k$ is not chosen via (31), we shall refer to a feedback $k$ that satisfies the pointwise condition (32) (for some rate function $W$ ) as a steepest descent feedback (induced by $V$ ). The value of $k(0)$ is irrelevant; we may set it equal to any point in $U$.

Theorem 20. A steepest descent feedback $k$ stabilizes the system in the sample-and-hold sense.

Proof. For ease of exposition, we shall suppose that $V$ (on $\mathbb{R}^{n}$ ) and $\nabla V$ (on $\left.\mathbb{R}^{n} \backslash\{0\}\right)$ are locally Lipschitz rather than merely continuous (otherwise, the argument is carried out with moduli of continuity). We also restrict attention to uniform partitions.

Let $B(0, R)$ and $B(0, r)$ be the initial values and target set under consideration. The properties of $V$ imply the existence of positive numbers $e<E$ such that

$$
\{x: V(x) \leq e\} \subset B(0, r),\{x: V(x) \leq E\} \supset B(0, R) .
$$

Fix $E^{\prime}>E$. There exist positive constants $K, L, M$ such that, for all $x, y$ in the compact set $\left\{x: V(x) \leq E^{\prime}\right\}$ and $u \in U$, we have

$$
\begin{aligned}
|V(x)-V(y)| \leq L|x-y|,|f(x, u)| & \leq M, \\
|f(x, u)-f(y, u)| & \leq K|x-y| .
\end{aligned}
$$

Now pick $e^{\prime}$ and $e^{\prime \prime}$ so that $0<e^{\prime \prime}<e^{\prime}<e$, and set

$$
X:=\left\{x: e^{\prime \prime} \leq V(x) \leq E^{\prime}\right\} .
$$

Then there exist constants $N$ and $\omega>0$ such that

$$
|\nabla V(x)-\nabla V(y)| \leq N|x-y|, W(x) \geq \omega \forall x, y \in X .
$$

We now give ourselves any uniform partition $\pi$ of diameter $\delta \in(0,1)$ such that

$$
\begin{array}{r}
\delta L M<\min \left\{e-e^{\prime}, e^{\prime}-e^{\prime \prime}, E^{\prime}-E\right\}, \\
\quad \delta(L K+M N) M<\omega / 2 .
\end{array}
$$

The reasons for these choices will emerge clearly below.

Now let $x_{0}$ be any point in $B(0, R)$, and proceed to implement the feedback $k$ via the partition $\pi$. On the first time interval $\left[t_{0}, t_{1}\right]$ the trajectory $x_{\pi}$ corresponding to $k$ (which we shall denote simply $x$ ) is generated by the differential equation

$$
x^{\prime}(t)=f\left(x(t), k\left(x_{0}\right)\right), x\left(t_{0}\right)=x_{0}, t_{0} \leq t \leq t_{1} .
$$

The solution to this differential equation exists on some interval of positive length, and is unique because $f$ is locally Lipschitz in the state variable. If the solution fails to exist on the entire interval, it is because blowup has occurred. Then there exists a first $\tau \in\left(t_{0}, t_{1}\right]$ for which $V(x(\tau))=E^{\prime}$. On the interval $\left[t_{0}, \tau\right)$, the Lipschitz constant $L$ of (33) is valid, as well as the bound $M$, whence

$V(x(t)) \leq V\left(x_{0}\right)+L\left|x(t)-x_{0}\right| \leq E+\delta L M \quad \forall t \in\left[t_{0}, \tau\right)$. But then

$$
V(x(\tau)) \leq E+\delta L M<E^{\prime}
$$

by (35), a contradiction. It follows that blow-up cannot occur, and that the solution of the differential equation exists on the entire interval $\left[t_{0}, t_{1}\right]$ and satisfies $V(x(t))<$ $E^{\prime}$ on that interval.

Case 1. $V\left(x_{0}\right) \leq e^{\prime}$. It follows then from $\delta L M<e-e^{\prime}$ (see (35)) that we have $V(x(t))<e \quad \forall t \in\left[t_{0}, t_{1}\right]$.

Case 2. $e^{\prime}<V\left(x_{0}\right)$. Now we have $x_{0} \in X$ and

$$
\left\langle\nabla V\left(x_{0}\right), f\left(x_{0}, k\left(x_{0}\right)\right\rangle<-\omega\right.
$$

from (34), and from the way $k\left(x_{0}\right)$ is defined (see (32)). Let $t \in\left(t_{0}, t_{1}\right]$; then, at least while $x(t)$ remains in the set $X$, we can argue as follows:

$$
V(x(t))-V\left(x\left(t_{0}\right)\right)=\left\langle\nabla V\left(x\left(t^{*}\right)\right), x^{\prime}\left(t^{*}\right)\right\rangle\left(t-t_{0}\right)
$$

(by the Mean Value Theorem, for some $t^{*} \in(0, t)$ )

$$
\begin{aligned}
& =\left\langle\nabla V\left(x\left(t^{*}\right)\right), f\left(x\left(t^{*}\right), k\left(t_{0}\right)\right)\right\rangle\left(t-t_{0}\right) \\
& =\left\langle\nabla V\left(x\left(t_{0}\right)\right), f\left(x\left(t_{0}\right), k\left(t_{0}\right)\right)\right\rangle\left(t-t_{0}\right) \\
& +\left\langle\nabla V\left(x\left(t_{0}\right)\right), f\left(x\left(t^{*}\right), k\left(t_{0}\right)\right)-f\left(x\left(t_{0}\right), k\left(t_{0}\right)\right)\right\rangle\left(t-t_{0}\right) \\
& +\left\langle\nabla V\left(x\left(t^{*}\right)\right)-\nabla V\left(x\left(t_{0}\right)\right), f\left(x\left(t^{*}\right), k\left(t_{0}\right)\right)\right\rangle\left(t-t_{0}\right) \\
& \leq\left\langle\nabla V\left(x\left(t_{0}\right)\right), f\left(x\left(t_{0}\right), k\left(t_{0}\right)\right)\right\rangle\left(t-t_{0}\right) \\
& +L K\left|x\left(t^{*}\right)-x_{0}\right|\left(t-t_{0}\right)+N M\left|x\left(t^{*}\right)-x_{0}\right|\left(t-t_{0}\right)
\end{aligned}
$$

(in view of (33) and (34))

$$
\leq-\omega\left(t-t_{0}\right)+L K M \delta\left(t-t_{0}\right)+M^{2} N \delta\left(t-t_{0}\right)
$$

(by the way $k$ is defined)

$$
\begin{aligned}
& =\{-\omega+\delta(L K+M N) M\}\left(t-t_{0}\right) \\
& \leq-(\omega / 2)\left(t-t_{0}\right), \text { by }(35) .
\end{aligned}
$$

Thus the value of $V$ has decreased. It follows from this (together with the inequality $\delta L M<e^{\prime}-e^{\prime \prime}$ provided by (35)) that $x(t)$ remains in $X$ throughout $\left[t_{0}, t_{1}\right]$, so that the estimates above apply.

To summarize, we have in Case 2 the following decrease property:

$$
V(x(t))-V\left(x\left(t_{0}\right)\right) \leq-(\omega / 2)\left(t-t_{0}\right) \quad \forall t \in\left[t_{0}, t_{1}\right] .
$$

It follows that, in either case, we have $V(x(t)) \leq E$ for $t \in\left[t_{0}, t_{1}\right]$, and in particular $V\left(x_{1}\right) \leq E$, where $x_{1}:=x\left(t_{1}\right)$ is the next node in the implementation scheme.

We now repeat the procedure on the next interval $\left[t_{1}, t_{2}\right]$, but using the constant control value $k\left(x_{1}\right)$. Precisely the same arguments as above apply to this and to all subsequent steps: either we are at a node $x_{i}$ for which $V\left(x_{i}\right) \leq e^{\prime}$ (Case 1), or else $V(x(t))$ continues to decrease at a rate of at least $\omega / 2$ (Case 2 ).

Since $V$ is nonnegative, the case of continued decrease cannot persist indefinitely. Let $x_{J}(J \geq 0)$ be the first node satisfying $V\left(x_{J}\right) \leq e^{\prime}$. If $J>0$, then

$$
\begin{aligned}
e^{\prime}<V\left(x_{J-1}\right) & \leq V\left(x_{0}\right)-(\omega / 2)\left(t_{J-1}-t_{0}\right) \\
& =V\left(x_{0}\right)-(\omega / 2)(J-1) \delta,
\end{aligned}
$$

whence

$$
\begin{aligned}
& \qquad(\omega / 2)(J-1) \delta<V\left(x_{0}\right)-e^{\prime} \leq E-e^{\prime}, \\
& \text { and so } \\
& J \delta<2\left(E-e^{\prime}\right) / \omega+\delta \leq 2\left(E-e^{\prime}\right) / \omega+1=: T,
\end{aligned}
$$

which provides a uniform upper bound $T$ independent of $\delta$ for the time $J \delta$ required to attain the condition $V\left(x_{J}\right) \leq e^{\prime}$. Once this condition is satisfied, the above 
analysis shows that for all $t \geq t_{J}$, we have $V(x(t)<e$, which implies $x(t) \in B(0, r)$. Since for all $t \geq 0$ the trajectory $x$ satisfies $V(x(t)) \leq E$, and since

$$
\{x: V(x) \leq E\} \subset B(0, R),
$$

there exists $C$ depending only on $R$ such that $|x(t)| \leq$ $C \forall t \geq 0$. This completes the proof that the required stabilization takes place.

Remark. It is clear that the proof essentially deals with the stabilization of level sets of $V$, and could be framed locally in these terms. Specifically, it shows that a steepest descent feedback stabilizes the set $\{x: V(x) \leq E\}$ to the set $\{x: V(x) \leq e\}$ under the following reduced requirements on $V$ (say): $V$ is continuous and nonnegative; the level set $\{x: V(x) \leq E\}$ is compact; $V$ is smooth in a neighborhood $\Omega$ of the set

$$
\{x: e \leq V(x) \leq E\} ;
$$

$V$ satisfies infinitesimal decrease in $\Omega$. Further, we observe that the value of $k(x)$ satisfying the decrease condition (32) could, in principle, be calculated online, and that the choice of such a value could be different in subsequent evaluations of $k(x)$

Example. Let $h: \mathbb{R} \rightarrow \mathbb{R}$ be a locally Lipschitz function satisfying $x h(x)>0 \quad \forall x \neq 0$. We consider the system

$$
\begin{aligned}
& x^{\prime}=y \\
& y^{\prime}=u|y|-h(x), \quad|u| \leq 4 .
\end{aligned}
$$

We claim that the feedback $k(x, y)=-4 \operatorname{sgn}(y)$ (where $\operatorname{sgn}(0)$ is taken to be any value in $[-1,+1])$ stabilizes the system in the sample-and-hold sense.

Proof. Let $H(x):=\int_{0}^{x} h(t) d t$. The function $V:=8 x^{2}+4 x y+y^{2}+2 H(x)=(2 x+y)^{2}+4 x^{2}+2 H(x)$ is $C^{1}$, positive definite and proper. The inner product

$$
\langle\nabla V(x, y), f(x, y, k(x, y))\rangle
$$

is given by $-4\left(y^{2}+x h(x)\right)$, for whatever value we assign to $\operatorname{sgn}(0)$. This establishes (32), for a suitable rate function $W$. The claim now follows from Theorem 20 .

\section{Semiconcave clf's}

Let $V$ be a Dini clf for the system $(*)$. Then, by definition, there exists for each nonzero point $x$ a value $u_{x} \in U$ such that

$$
d V\left(x ; f\left(x, u_{x}\right)\right)<-W(x) .
$$

It is tempting to define a feedback via $k(x):=u_{x}$. One might well expect (in view of the smooth case analyzed above) that this feedback would lead to trajectories along which $V$ decreases. However, such is not the case in general, as we now see.

Example. We take $n=2$, and we assume that for all $(x, y)$, the set $f(x, y, U)$ contains the four vectors

$$
v_{1}=(1,0), v_{2}=(-1,0), v_{3}=(0,1), v_{4}=(0,-1) .
$$

We set

$$
V(x, y):=|x-y|+2|y|
$$

a function which is locally Lipschitz, positive definite, and proper.

Let $(x, y)$ be any nonzero point. If $x \neq y$, then one of the unit vectors (admissible velocities) $v_{2}=(-1,0)$ or $v_{1}=(1,0)$ (depending on whether $x-y$ is positive or negative respectively) will give $d V(x, y ; v)=-1$. If $x=y$, then the unit vector $(0,-y /|y|)$ (that is, $v_{3}$ or $\left.v_{4}\right)$ has the same effect. It follows that $V$ is a Dini clf (with rate function $W \equiv 1$ ), and that the system is asymptotically controllable.

Now suppose we find ourselves at a point $(x, y)$ with $x=y-\epsilon$ for a small positive $\epsilon$. A direction which decreases $V$ (at rate 1 ) is given by $(1,0)$; a small step in that direction may bring us to the point $(y+\epsilon, y)$ (say). From this new point, a decrease direction is $(-1,0)$, and a small step brings us back to the starting value $(y-\epsilon, y)$. This can go on indefinitely, so a pointwise choice of a decrease direction (as in steepest descent) does not always succeed in driving the state to 0 , due to the possibility of dithering.

In this example, it is clear that a smooth CLF exists, for example $V(x, y)=\left(x^{2}+y^{2}\right) / 2$, with rate function $W(x, y)=\max \{|x|,|y|\}$. One can then consider the steepest descent feedback associated with this smooth $V$, which is easily calculated:

$$
k(x, y)= \begin{cases}-\operatorname{sgn}(x) & \text { if }|x|>|y| \\ -\operatorname{sgn}(y) & \text { if }|y|>|x|\end{cases}
$$

(with either choice when $|x|=|y|$ ).

Then $k$ stabilizes the system (by Theorem 20). But the point being made here is that the design of $k$ on the basis of a nonsmooth $V$ is a more subtle issue than merely using steepest descent.

We shall present several methods that deal with the problem: one requires that the clf be semiconcave (which is not the case in the last example), another will work by constructing a different clf through smoothing (when the original one in locally Lipschitz), and a third is based on selecting the descent direction in a more complex fashion than steepest descent.

Let $V$ be a semiconcave clf (that is, satisfying the property SC of Section 5 on $\mathbb{R}^{n} \backslash\{0\}$ ). We say that a feedback $k$ is of steepest descent type (for $V$ ) if, for some rate function $W$, we have

$$
d V(x ; f(x, k(x)))=V^{\prime}(x ; f(x, k(x)))<-W(x) \forall x \neq 0 .
$$

By Part (13) of Theorem 14, this is equivalent to:

$$
\begin{aligned}
& \forall x \neq 0, \exists \zeta \in \partial_{C} V(x) \text { such that } \\
& \qquad\langle\zeta, f(x, k(x))\rangle<-W(x) .
\end{aligned}
$$

Theorem 21. If $V$ is a semiconcave (proximal or Dini) clf, and if $k$ is a steepest descent feedback induced by $V$, then $k$ stabilizes the system in the sample-and-hold sense.

Proof. We proceed to sketch how to adapt the proof of Theorem 20. Let $e^{\prime}<e$ and $E^{\prime}>E$ be such that $V$ has property $\mathrm{SC}$ on the set

$$
X:=\left\{x: e^{\prime} \leq V(x) \leq E^{\prime}\right\} .
$$


We find constants $L, \sigma, \eta$ and $\rho$ as in Part (2) of Theorem 14. Then $K, L$ and $M$ satisfying (33) continue to exist. We maintain the choice of $\omega$ as in (34), but there is no $N$ as appears there, of course; the second inequality in (35) needs to be replaced.

The essential step remains the comparison of the values of $V$ at successive nodes, but condition $\mathrm{SC}$ is invoked as a substitute for the Mean Value Theorem. If $\delta \in(0,1)$ is sufficiently small (precisely: $\delta M<\rho$ ), successive nodes will lie within $\rho$ of one another. Let $\zeta_{0} \in \partial_{C} V\left(x_{0}\right)$ be such that

$$
V^{\prime}\left(x_{0} ; f\left(x_{0}, k\left(x_{0}\right)\right)\right)=\left\langle\zeta_{0}, f\left(x_{0}, k\left(x_{0}\right)\right)\right\rangle
$$

(see (38)). Then, between the first two nodes, and in Case 2 of the previous proof, we have:

$$
\begin{aligned}
& V(x(t))-V\left(x\left(t_{0}\right)\right) \\
& \leq\left\langle\zeta_{0}, x(t)-x\left(t_{0}\right)\right\rangle\left(t-t_{0}\right)+\sigma\left|x(t)-x\left(t_{0}\right)\right|^{1+\eta}
\end{aligned}
$$

(by Part (2) of Theorem 14; this is where SC is used)

$$
=\left\langle\zeta_{0}, f\left(x\left(t^{*}\right), k\left(t_{0}\right)\right)\right\rangle\left(t-t_{0}\right)+\sigma\left|x(t)-x\left(t_{0}\right)\right|^{1+\eta}
$$

(for some $t^{*} \in\left(t_{0}, t\right)$, by the Mean Value Theorem)

$$
\begin{aligned}
& \leq\left\langle\zeta_{0}, f\left(x\left(t_{0}\right), k\left(t_{0}\right)\right)\right\rangle\left(t-t_{0}\right) \\
& \quad+K L\left|x\left(t^{*}\right)-x\left(t_{0}\right)\right|\left(t-t_{0}\right)+\sigma\left[M\left(t-t_{0}\right)\right]^{1+\eta} \\
& \leq-\omega\left(t-t_{0}\right)+K L M \delta\left(t-t_{0}\right)+\sigma M^{1+\eta} \delta^{\eta}\left(t-t_{0}\right)
\end{aligned}
$$

(by the definition of $k\left(x_{0}\right)$ and choice of $\zeta_{0}$ )

$$
\begin{aligned}
& \leq\left\{-\omega+K L M \delta+\sigma M^{1+\eta} \delta^{\eta}\right\}\left(t-t_{0}\right) \\
& \leq-(\omega / 2)\left(t-t_{0}\right),
\end{aligned}
$$

if $K L M \delta+\sigma M^{1+\eta} \delta^{\eta}<\omega / 2$ : this is the replacement for the second part of (35). Thus a uniform decrease in the value of $V$ can be guaranteed by taking $\delta$ sufficiently small, and the proof proceeds as before.

Remark. The proof shows that a feedback $k$ defined by steepest descent stabilizes $\{V \leq E\}$ to $\{V \leq e\}$, provided that $V$ is continuous and positive definite, the level set $\{x: V(x) \leq E\}$ is compact, and $V$ satisfies both property $\mathrm{SC}$ and infinitesimal decrease on a neighborhood of the set $\{x: e \leq V(x) \leq E\}$.

Example. We showed earlier that the function $V_{1}$ given by (27) is a (Dini or proximal) clf for the nonholonomic integrator (14). In Section 5, we proved that $V_{1}$ is semiconcave. In view of Theorem 21, any steepest descent feedback $k$ induced by it will stabilize the system in the sampleand-hold sense; we proceed to calculate one explicitly. As before, we set

$$
\begin{aligned}
\sigma & :=\sqrt{x_{1}^{2}+x_{2}^{2}} \\
W(x) & :=2 \max \left\{|\sigma-| x_{3}||, \sigma\left|\sigma \operatorname{sgn}\left(x_{3}\right)-2 x_{3}\right|\right\},
\end{aligned}
$$

where $\operatorname{sgn}(0)$ is taken to be 0 .

Consider a point $x=\left(x_{1}, x_{2}, x_{3}\right) \neq 0$. When $\sigma>0$ and $x_{3} \neq 0$, the calculations of Section 4 show that we may achieve pointwise decrease of $V_{1}$ at rate $W(x)$ by taking $k(x)=\left\{\begin{aligned}\left(x_{1}, x_{2}\right) / \sigma & \text { if }\left|x_{3}\right|-\sigma \geq \sigma\left|\sigma \operatorname{sgn}\left(x_{3}\right)-2 x_{3}\right| \\ -\left(x_{1}, x_{2}\right) / \sigma & \text { if } \sigma-\left|x_{3}\right| \geq \sigma\left|\sigma \operatorname{sgn}\left(x_{3}\right)-2 x_{3}\right| \\ \left(x_{2},-x_{1}\right) / \sigma & \text { if } \sigma\left(2 x_{3}-\sigma \operatorname{sgn}\left(x_{3}\right)\right)>|\sigma-| x_{3}|| \\ -\left(x_{2},-x_{1}\right) / \sigma & \text { if } \sigma\left(\sigma \operatorname{sgn}\left(x_{3}\right)-2 x_{3}\right)>|\sigma-| x_{3}|| .\end{aligned}\right.$

When $\sigma=0$, then $x_{3} \neq 0$, and the choice $k(x)=(1,1) / \sqrt{2}$ yields

$$
d V_{1}\left(x ; f(x, k(x)) \leq-2\left|x_{3}\right| \leq-W(x) .\right.
$$

When $x_{3}=0$ (so $\sigma \neq 0$ ), the choice $k(x)=-\left(x_{1}, x_{2}\right) / \sigma$ yields

$$
d V_{1}(x ; f(x, k(x)) \leq-2 \sigma \leq-W(x) .
$$

Then $k(x)$ has been defined for all $x \neq 0$, and admits the rate function $W$; since $V_{1}$ is semiconcave, Theorem 21 assures us that $k$ stabilizes the system.

The function $V_{2}$ of (28) is another clf for the nonholonomic integrator. But since it is not semiconcave, it cannot serve as above to define a stabilizer. The question of how to design a stabilizing feedback if one has only a clf that fails to satisfy SC is the next topic, first in the case in which $V$ is locally Lipschitz, and subsequently when $V$ is merely continuous.

\section{Locally Lipschitz clf's}

The purpose of this section is to show how practical semiglobal stabilizing feedbacks can be constructed from a Dini clf that is not known to be semiconcave, but is locally Lipschitz. As noted earlier, the pointwise condition $d V(x ; f(x, k(x)))<-W(x)$ does not guarantee stabilization when $V$ fails to be semiconcave (or smooth).

For a positive parameter $\lambda$, we define

$$
V_{\lambda}(x):=\min _{z \in \mathbb{R}^{n}}\left\{V(z)+(\lambda / 2)|x-z|^{2}\right\} .
$$

This is sometimes referred to as a quadratic inf-convolution (of $V$ ). Clearly, we have $0 \leq V_{\lambda} \leq V$. It can be shown (see Clarke et al. (1998)) that $\lim _{\lambda \rightarrow \infty} V_{\lambda}(x)=V(x)$ for each $x$. More precisely, one shows that given any compact set $X$ and positive $\epsilon$, there exists $\lambda$ sufficiently large so that $\left|V_{\lambda}(x)-V(x)\right|<\epsilon \quad \forall x \in X$.

We see therefore that $V_{\lambda}$ approximates $V$, in a way that improves as $\lambda$ is taken larger. The point of the calculations below is to show that, for $\lambda$ sufficiently large, $V_{\lambda}$ satisfies infinitesimal decrease as well as property SC on a neighborhood of the set

$$
S:=\{x: e \leq V(x) \leq E\} .
$$

This means that it can be used to define a steepest descent feedback for practical semiglobal stabilization, as we did earlier in the semiconcave case.

Note that the minimum defining $V_{\lambda}(x)$ is always attained by at least one point $z_{x}$. By definition of $V_{\lambda}$, we have

$$
V\left(z_{x}\right)+\lambda\left|x-z_{x}\right|^{2} / 2 \leq V(x) .
$$

If $x$ is restricted to a bounded set $X$, and if $M_{V}$ is an upper bound for $V$ on the set $X$, then, since $V \geq 0$, this inequality yields $\lambda\left|x-z_{x}\right|^{2} / 2 \leq M_{V}$. It is a consequence 
of this observation that if $\mu>0$ is given, then, for all $\lambda$ sufficiently large, we have $\left|x-z_{x}\right|<\mu \forall x \in X$.

It now follows from this, together with Part (11) of Theorem 14 (invoked locally on bounded sets), that $V_{\lambda}$ satisfies SC at each point of $\mathbb{R}^{n}$. (In order to invoke the theorem, we observe that the minimum defining $V_{\lambda}$ is equivalent to a countable infimum in which $z$ is restricted to having rational coordinates.)

With the above facts in mind, we now suppose that $V$ satisfies infinitesimal decrease (in the Dini sense) on a neighborhood $\Omega$ of the closed set $S:=\{x: e \leq V(x) \leq E\}$, which we assume compact. We proceed to show how to use $V_{\lambda}$ to define a feedback stabilizing the level set $\{V \leq E\}$ to the level set $\{V \leq e\}$.

Fix $\omega>0$ so that $W(x)>\omega \forall x \in \Omega$. Let $\Omega^{\prime}$ be an open set satisfying

$$
S \subset \Omega^{\prime} \subset \overline{\Omega^{\prime}} \subset \Omega,
$$

and fix $e^{\prime} \in(0, e)$ so that $\left\{x: e^{\prime} \leq V(x) \leq E\right\} \subset \Omega^{\prime}$. Then, for $\lambda$ sufficiently large, we have

$$
\begin{aligned}
\left\{x: V_{\lambda}(x) \leq e^{\prime}\right\} \subset\{x: V(x) \leq e\}, & \\
& \left\{x: V_{\lambda}(x) \leq E\right\} \supset\{x: V(x) \leq E\} .
\end{aligned}
$$

(The second inclusion here is in fact automatic, for any $\lambda$.) We also have that, for any point $x \in \Omega^{\prime}$, any point $z_{x}$ at which the minimum defining $V_{\lambda}(x)$ is attained satisfies $z_{x} \in \Omega$. For any such $x$ and $z_{x}$, the minimization implies

$$
d V\left(z_{x} ; v\right)+\lambda\left\langle z_{x}-x, v\right\rangle \geq 0 \quad \forall v \in \mathbb{R}^{n} .
$$

Now choose any $u_{x} \in U$ satisfying

$$
d V\left(z_{x} ; f\left(z_{x}, u_{x}\right)\right)<-\omega
$$

this is possible because $V$ satisfies infinitesimal decrease in $\Omega$. Set $f_{z}:=f\left(z_{x}, u_{x}\right)$ and $f:=f\left(x, u_{x}\right)$.

Lemma. If $\lambda$ is sufficiently large, we have

$$
d V\left(z_{x} ; f\right)<-\omega / 2 .
$$

Proof. Let $K_{V}$ be a Lipschitz constant for $V$ on the relevant set. Let $d V\left(z_{x} ; f_{z}\right)$ be realized by the sequence $t_{i}$ :

$$
d V\left(z_{x} ; f_{z}\right)=\lim _{i} \frac{V\left(z_{x}+t_{i} f_{z}\right)-V\left(z_{x}\right)}{t_{i}}<-\omega .
$$

Then

$$
\begin{aligned}
d V\left(z_{x} ; f\right) \leq & \liminf _{i} \frac{V\left(z_{x}+t_{i} f\right)-V\left(z_{x}\right)}{t_{i}} \\
\leq & \limsup _{i} \frac{V\left(z_{x}+t_{i} f\right)-V\left(z_{x}+t_{i} f_{z}\right)}{t_{i}} \\
& +\liminf _{i} \frac{V\left(z_{x}+t_{i} f_{z}\right)-V\left(z_{x}\right)}{t_{i}} \\
\leq & K_{V}\left|f-f_{z}\right|+d V\left(z_{x} ; f_{z}\right) \leq-\omega / 2
\end{aligned}
$$

if $K_{V}\left|f-f_{z}\right|<\omega / 2$. But this holds when $\lambda$ is sufficiently large, since $\left|f-f_{z}\right| \leq K_{f}\left|x-z_{x}\right|$, where $K_{f}$ is a suitable Lipschitz constant for $f$.

Now (41) and the lemma imply $\lambda\left\langle x-z_{x}, f\right\rangle<-\omega / 2$. Then

$$
\begin{aligned}
& \frac{V_{\lambda}(x+t f)-V_{\lambda}(x)}{t} \\
& \leq \frac{V\left(z_{x}\right)+\lambda\left|x+t f-z_{x}\right|^{2} / 2-V\left(z_{x}\right)-\lambda\left|x-z_{x}\right|^{2} / 2}{t} \\
& =\lambda\left\langle x-z_{x}, f\right\rangle+\lambda t|f|^{2} / 2,
\end{aligned}
$$

whence $d V_{\lambda}(x ; f)<-\omega / 2$.

This shows that $V_{\lambda}$ satisfies infinitesimal decrease on the set $\Omega^{\prime}$. Since it also has property SC, Theorem 21 (see the remark after the theorem) assures us that the steepest descent feedback $k$ (for $V_{\lambda}$ ) stabilizes $\left\{V_{\lambda} \leq E\right\}$ to $\left\{V_{\lambda} \leq\right.$ $\left.e^{\prime}\right\}$. By (40), this implies that the set $\{V \leq E\}$ is stabilized to $\{V \leq e\}$. We have proved:

Theorem 22. Let $V$ be locally Lipschitz and nonnegative. Suppose that the set $\{x: V(x) \leq E\}$ is compact, and that $V$ satisfies infinitesimal decrease (in the Dini sense, for some rate function) on a neighborhood of the set

$$
\{x: e \leq V(x) \leq E\} .
$$

Then, for $\lambda$ sufficiently large, a steepest descent feedback induced by $V_{\lambda}$ stabilizes $\{V \leq E\}$ to $\{V \leq e\}$.

Note that in contrast to the smooth or semiconcave cases examined previously, the stabilizing feedback is no longer defined via $V$, but rather via $V_{\lambda}$. Furthermore, the $\lambda$ must be sufficiently large in a way that depends on $r$ and $R$, and hence on the desired degree of practical semiglobal stabilization. Thus, in contrast to the earlier smooth and semiconcave cases, we have not constructed here a single feedback that stabilizes the system in the sample-and-hold sense, although Theorem 19 assures us that one exists. (It can be constructed only at the expense of considerably more technical difficulty.)

Example. We return to the system (30). We had found a function

$$
V(x, y)=|| y|-1|+|x|
$$

which is a clf with respect to a subset $\mathcal{T}$ of $B(0,1)$. It is easy to see that $V$ is locally Lipschitz, but that it does not satisfy property SC on $\mathbb{R}^{n} \backslash \mathcal{T}$. (Consider the point $(0,2)$, for example, where $V$ has a 'convex corner' and cannot therefore be semiconcave.) This accounts for the fact that the feedback associated to the field of trajectories that generated $V$ (see Fig. 6) fails to stabilize the system to $\mathcal{T}$ in the sample-and-hold sense: dithering may occur at points on the set $|y|=1$, or at points on the $y$-axis.

To find a suitable feedback by Theorem 22, we are led to calculate the function

$V_{\lambda}(x, y)=\min _{\alpha, \beta}|\alpha|+|| \beta|-1|+(\lambda / 2)\left(|x-\alpha|^{2}+|y-\beta|^{2}\right)$

together with its steepest descent feedback $k$. Restricting attention to the first quadrant for ease of exposition, we obtain different expressions for $V_{\lambda}$, corresponding to whether $x$ is to the left or right of $\lambda^{-1}$, and depending also upon where $y$ is situated relative to $1-\lambda^{-1}$ and $1+\lambda^{-1}$ (thus, six different cases).

The corresponding values of the steepest descent feedback induced by $V_{\lambda}$ are then easily found. The resulting directions of movement are indicated on Fig. 8, except in the 
domain that contains the point $(0,1): V_{\lambda}$ does not satisfy infinitesimal decrease there.

Stabilization no longer leads to the point $(0,1)$, as the field of trajectories does, but to a neighborhood of that point, one which can be made arbitrarily small by taking $\lambda$ sufficiently large.

We observe that, in contrast to the initial field of trajectories (see Fig. 6), the feedback induced by $V_{\lambda}$ admits a zone around $y=1$ with

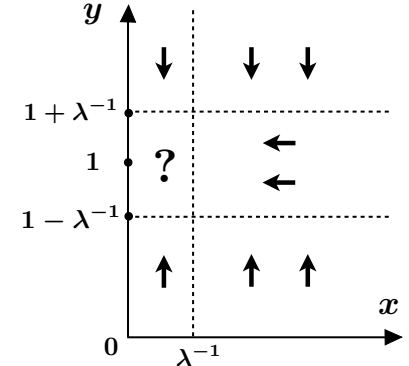

Fig. 8. A stabilization strategy positive thickness in which the trajectories move to the left. This feature, which is reminiscent of a technique sometimes used in sliding-mode control to reduce dither, is an automatic byproduct of our approach. In effect, it is why sample-and-hold stabilization takes place.

\section{Continuous clf's}

Let $V$ be a proximal clf that is merely continuous, without any of the extra assumptions introduced above (smooth, semiconcave, or locally Lipschitz). (This includes the case of a merely continuous Dini clf, in view of Prop. 11.) We now show how to use $V$ to define stabilizing feedbacks for practical semiglobal stabilization.

The definition of the feedback amounts to a conceptual algorithm. We design the feedback $k_{\lambda}(x)$ as follows, for any $x$ :

Find a point $z_{x}$ minimizing $z \mapsto V(z)+\lambda|x-z|^{2} / 2$, then select $k_{\lambda}(x) \in \operatorname{argmin}_{u \in U}\left\langle x-z_{x}, f\left(z_{x}, u\right)\right\rangle$.

Note that this definition requires more computation than does a steepest descent feedback.

Theorem 23. Let $\{V \leq E\}$ and $\{V \leq e\}$ be given level sets of a proximal clf, where $0<e<E$. Then, for $\lambda$ sufficiently large, the feedback $k_{\lambda}$ stabilizes $\{V \leq E\}$ to $\{V \leq e\}$.

Proof. (Sketch) We define $V_{\lambda}$ by (39) as before. Recall that we can choose $\lambda$ sufficiently large as in the proof of Theorem 22 so that the level sets of $V$ are closely approximated by corresponding ones of $V_{\lambda}$, and so that $z_{x}$ is $\mu$-close to $x$ for all $x$ in a bounded set $\Omega$ containing those level sets. By definition, we have

$$
V\left(z_{\lambda}\right)+\lambda\left|x-z_{x}\right|^{2} / 2 \leq V(x),
$$

$$
\lambda\left|x-z_{x}\right|^{2} \leq 2\left(V(x)-V\left(z_{\lambda}\right)\right) \leq 2 m(\mu),
$$

where $m$ is a modulus of continuity for $V$ on $\Omega$.

It follows from the definition of $z_{x}$ that

$$
\zeta:=\lambda\left(x-z_{x}\right) \in \partial_{P} V\left(z_{x}\right) .
$$

Because $V$ is a proximal clf, we deduce that, for some positive $\omega$, and for all $x \in \Omega$,

$$
\min _{u \in U}\left\langle\zeta, f\left(z_{x}, u\right)\right\rangle<-\omega .
$$

Then, setting $f:=f\left(x, k_{\lambda}(x)\right)$, we have, by definition of $k_{\lambda}$, and by (42):

$$
\begin{aligned}
\langle\zeta, f\rangle & =\min _{u \in U}\left\langle\lambda\left(x-z_{x}\right), f(x, u)\right\rangle \\
& \leq \min _{u \in U}\left\langle\lambda\left(x-z_{x}\right), f\left(z_{x}, u\right)\right\rangle+K_{f} \lambda\left|x-z_{x}\right|^{2} \\
& <-\omega+2 K_{f} m(\mu)<-2 \omega / 3,
\end{aligned}
$$

if $\mu$ has been chosen small enough. We now derive, as in the proof of Theorem 22, the estimate

$$
\begin{aligned}
V_{\lambda}(x+t f)-V_{\lambda}(x) & \leq t \lambda\left\langle x-z_{x}, f\right\rangle+\lambda t^{2}|f|^{2} / 2 \\
& \leq-t \omega / 3,
\end{aligned}
$$

if $t \leq \delta$ for a mesh size $\delta$ sufficiently small. As in previous arguments, this provides the decrease between nodes that guarantees the stabilization of level sets of $V_{\lambda}$ (and hence of $V$ ).

The minimal-time function $T(\cdot)$ found for the double integrator (8) is an example of a merely continuous clf, with none of the additional regularity used earlier. It can be shown, however, by an ad hoc argument, that a pointwise steepest descent feedback induced by $T$ does nonetheless stabilize the system in this case (in contrast to the example (30), where this fails).

We remark that there also exists a smooth clf for the double integrator $(8)$. We prove this as follows. Define a multifunction $F(x, y)$ to equal $\{(y,-1)\}$ to the right of the switching curve $S,\{(y,+1)\}$ to the left, and $\{y\} \times[-1,1]$ on $S$. Then we can show that the trajectories of $F$ are exactly the minimal-time ones, and so $\left(x^{\prime}, y^{\prime}\right) \in F(x, y)$ is strongly stable. Because $F$ is relaxed and has closed graph, it follows from Theorem 1 that $F$ admits a smooth clf $V$ in the strong sense. Then $V$ is a smooth clf for the system (8). As a consequence of this, we also deduce (by Theorem 5) that the system admits a continuous stabilizing feedback (for which we have no explicit expression).

\section{State constraints, optimal feedbacks}

There are situations in which the state $x$ is naturally constrained to lie in a given closed set $S$, so that in steering the state to the origin, we must respect the condition $x(t) \in S$, referred to as a (unilateral) state constraint. The same questions arise as in the unconstrained case: is the possibility of doing this in the open-loop sense characterized by some kind of Lyapunov function, and would such a function lead to the design of a stabilizing feedback that respects the state constraint? The more challenging case is that in which the origin lies on the boundary of $S$, but the case in which 0 lies in the interior of $S$ is also of interest, since it serves in particular to localize around the origin the global, state-constraint-free situation that has been the focus of this article.

An important consideration in dealing with state constraints is to identify a class of sets $S$ for which meaningful results can be obtained. In Clarke and Stern (2003, 2005), the methods described above are extended to the case of state constraints specified by a set $S$ which is wedged (see Clarke et al. (1998)). This rather large class of sets includes smooth manifolds with boundaries and convex bodies (as well as their closed complements). 
A further hypothesis is made regarding the consistency of the state constraint with the dynamics of the system: for every nonzero vector $\zeta$ in the normal cone co $N_{S}^{L}(x)$ to a point $x \in \operatorname{bdry} S$ (see Section 3), there exists $u \in U$ such that $\langle f(x, u), \zeta\rangle<0$. Thus an 'inward-pointing' velocity vector is always available.

Under these conditions, and in terms of suitably-defined extensions to the state-constrained case of the underlying definitions, one can prove the equivalence between controllability, feedback stabilization, and the existence of (locally Lipschitz or semiconcave) control-Lyapunov functions.

A different issue arises in optimal control: that of designing feedbacks which generate optimal (or near-optimal) trajectories. Results in this vein are described in Nobakhtian and Stern (2000), and in Clarke et al. (2002) for the case in which the optimal control problem features state constraints. The methodology is much the same as that of Theorem 23, but with the clf's replaced by value functions.

\section{Robustness}

The use of sample-and-hold implementation allows a rigorous and detailed error analysis of the feedbacks designed above, encompassing many possible types of error. We give here only a brief introduction to the topic (but see also Section 10). We refer to Clarke et al. (2000), Ledyaev and Sontag (1999), and Sontag (1999) for details.

To illustrate, suppose that in applying the feedback $k$ at the node $x_{i}$ we have, on the partition interval $\left[t_{i}, t_{i+1}\right]$, not the ideal differential equation

$$
x^{\prime}(t)=f\left(x(t), k\left(x_{i}\right)\right)
$$

considered previously, but rather

$$
x^{\prime}(t)=f\left(x(t)+e_{1}, k\left(x_{i}+m\right)+e_{2}\right)+e_{3},
$$

where $e_{1}, e_{2}, e_{3}$ reflect imprecision in applying (or modeling) $f$, and where $m$ is measurement error in sampling the state at the node $x_{i}$. Then the fact that $f$ is continuous allows us to write

$$
x^{\prime}(t)=f\left(x(t), k\left(x_{i}+m\right)\right)+a,
$$

where $a$ is small when $e_{1}, e_{2}, e_{3}$ are small. We call $a$ the actuator error. Other types of imprecision can be reduced to actuator error as well, such as delay in applying the feedback. (This is discussed in Clarke and Vinter (2009).)

Note, however, that $m$ cannot be reduced to $a$ in this way, since $k$ is not continuous. In fact, measurement error $m$ and actuator error $a$ represent fundamentally different types of error, leading to different robustness results.

Roughly speaking, sample-and-hold implementation of a Lyapunov-based steepest descent feedback is always robust to actuator error, in the sense that the underlying feedback still stabilizes the system, within a tolerance that is proportional to the size of the error. Without going into full detail, let us examine a typical argument leading to such a conclusion (we take measurement error $m=0$ ).

In the proof of Theorem 20, we wish to show that decrease of $V$ between nodes (that is, the calculation of Case 2) continues to hold when $f$ is replaced by $f+a$, provided that $|a|$ is sufficiently small. The initial estimate in the original proof becomes

$$
V(x(t))-V\left(x\left(t_{0}\right)\right)=\left\langle\nabla V\left(x\left(t^{*}\right)\right), x^{\prime}\left(t^{*}\right)\right\rangle\left(t-t_{0}\right)
$$

(by the Mean Value Theorem, for some $t^{*} \in(0, t)$ )

$$
=\left\langle\nabla V\left(x\left(t^{*}\right)\right), f\left(x\left(t^{*}\right), k\left(t_{0}\right)\right)+a\right\rangle\left(t-t_{0}\right) .
$$

We observe that this last term is bounded above by

$$
\left\langle\nabla V\left(x\left(t^{*}\right)\right), f\left(x\left(t^{*}\right), k\left(t_{0}\right)\right)\right\rangle\left(t-t_{0}\right)+N|a|\left(t-t_{0}\right) .
$$

After the same series of estimates as before, we now obtain, at the end, the following upper bound on the decrease between nodes:

$$
\{-\omega+\delta(L K+M N) M+N|a|\}\left(t-t_{0}\right) .
$$

(Note the new term involving $a$.) Overall, this can still be guaranteed to be negative (for $\delta$ sufficiently small), provided that $|a|<\omega / N$. Thus, decrease of $V$ between nodes (and hence, stabilization) continues until the decrease rate $\omega$ becomes too small to deal with the actuator error.

We summarize to this point by saying that actuator error simply degrades performance proportionally to its size; we retain a degree of practical stabilization.

Consider now the possibility of state measurement error in implementing the feedback law: we are at $x$, but measure the state as $x+m$, and therefore apply the control $k(x+m)$ instead of the correct value $k(x)$. When $k$ is continuous, then small $m$ leads to a small effect (which can be reduced to actuator error, as above). In the case of discontinuous feedback, the effect of measurement error is more subtle, and generally harder to deal with.

It is a fact that the (generally discontinuous) feedback laws constructed above do possess a relative robustness property: if, in the sample-and-hold implementation, the measurement error is at most of the same order of magnitude as the partition diameter, then practical stabilization is obtained. To put this another way, the step size may have to be big enough relative to the potential errors (to avoid dithering, for example).

This is different from the preceding actuator error analysis, where, once the size of the error is bounded, all sufficiently fine partitions give rise to stabilization (up to a certain point, depending on the bound). Now, the bound on the measurement error is a function of the partition diameter.

There is another, stronger type of robustness (we call it absolute robustness), in which the presence of small measurement errors preserves practical stabilization independently of the step size. Ledyaev and Sontag (1999) have shown that there exists an absolutely robust stabilizing feedback for the system if and only if it admits a smooth clf. This, then, is an advantage that such systems have.

To illustrate, recall that the nonholonomic integrator (14) and the system (17) both fail to be stabilizable by continuous feedback; both admit discontinuous stabilizers. The nonholonomic integrator does not admit a smooth clf, and hence fails to admit an absolutely robust stabilizing feedback. The system (17), on the other hand, does so. We remark that to recover absolute robustness, one can turn to dynamic feedback; see Ledyaev and Vinter (2010). 
Regular and essentially stabilizing feedbacks

In view of the fact that an asymptotically controllable system need not admit a continuous stabilizing feedback, the question arises of the extent to which the discontinuities of a stabilizer can be minimized. L. Rifford has shown (nonconstructively) that, for systems affine in the control, there exists a stabilizing feedback whose discontinuities form a set of measure zero. Moreover, the discontinuity set is repulsive for the trajectories generated by the feedback: the trajectories lie in that set at most initially. In the case of planar systems, Rifford has classified the types of discontinuity that must occur in stabilizing feedbacks. He has also proved that there exists a smooth feedback which almost stabilizes the system (that is, from almost all initial values).

We use the rest of our time together to consider two settings in which discontinuous feedbacks play a central role: feedback blending, and sliding modes.

\section{FEEDBACK BLENDING}

It may happen that we have two feedbacks on hand for the system $(*)$, one that stabilizes effectively near the origin, the other that drives distant points to a neighborhood of 0 . This raises the issue of how to make the transition from one to the other. If the two feedbacks are continuous, one may want the new combined feedback to be continuous. This is decidedly problematic.

In the context of sample-and-hold implementation of feedbacks defined by means of a clf, however, continuity is irrelevant, and certain natural constructions become possible.

Let $V_{0}$ be a clf for the system (in the smooth, Dini, or proximal sense) that drives all points in the level set $\left\{x: V_{0}(x) \leq \Delta_{0}\right\}$ to the origin, and let $k_{0}$ be a stabilizing feedback associated to it by one of the methods described earlier.

Now let $V_{1}$ be a clf inducing practical stabilization to the target set $\left\{x: V_{1}(x) \leq \delta_{1}\right\}$, and $k_{1}$ its associated feedback. We wish to blend $k_{0}$ and $k_{1}$ into a single feedback $k$ so that $k=k_{1}$ for large $x$, and so that $k_{0}$ takes over near the origin: $k=k_{0}$ in a neighborhood of 0 .

The motivation for this might be that $k_{0}$ is a feedback with desirable regularity properties, but which fails to have values in the set $U$ when $x$ is large. For example, $k_{0}(x)=K x$ might be a linear feedback valid near the origin, but which satisfies $k_{0}(x) \in U$ only when $x$ is sufficiently small (since $U$ is compact). $V_{1}$, for its part, might be a clf giving only practical stabilization, perhaps obtained by the methods of Section 6 .

In order to hope to succeed in this endeavor, it is clear that we must have at least

$$
\left\{x: V_{1}(x) \leq \delta_{1}\right\} \subset\left\{x: V_{0}(x) \leq \Delta_{0}\right\} .
$$

We now describe two different approaches to the issue.

\section{A hybrid transition}

Let us suppose that (43) holds in a stronger sense, with overlap: for some $\eta>0$ we have

$$
\left\{x: V_{1}(x) \leq \delta_{1}+\eta\right\} \subset\left\{x: V_{0}(x)<\Delta_{0}\right\} .
$$

Then we introduce a modified sample-and-hold implementation scheme as follows: from a given initial state value $x_{0}$, if $V_{0}\left(x_{0}\right)<\Delta_{0}$, the feedback $k_{0}$ is used exclusively: stabilization to the origin results. If $V_{0}\left(x_{0}\right) \geq \Delta_{0}$, then $k_{1}$ is applied in the initial phase of sample-and-hold. Subsequently, there must be a first node $x_{i}$ for which $V_{1}\left(x_{i}\right)<\delta_{1}+\eta / 2$; thus $V_{0}\left(x_{i}\right)<\Delta_{0}$, in light of (44). Starting at that node, we apply $k_{0}$ thereafter.

All initial conditions $x_{0}$ are stabilized to the origin in this manner. Note, however, that there is a new hybrid element to the stabilization scheme, since a switch of feedbacks occurs along the way, and the values of $V_{0}$ and $V_{1}$ must be monitored until the switch occurs. We describe now another approach that brings us back to the case of a steepest descent feedback induced by a single clf.

(Remark: in the first phase of this procedure, which aims for the set $\left\{x: V(x)<\delta_{1}+\eta / 2\right\}$, the thin set fallacy is avoided, since the (open) set in question is 'fat'.)

A new clf

We assume that $V_{0}$ is semiconcave, and satisfies infinitesimal decrease (see (38)) on the set $\left\{x: V_{0}(x) \leq \Delta_{0}\right\}$. Concerning $V_{1}$, we assume that, on the complement of the set $\left\{x: V_{1}(x) \leq \delta_{1}\right\}$, it satisfies infinitesimal decrease and is semiconcave (that is, satisfies property $\mathrm{SC}$ of Section 5 at each point).

The hypothesis (43) is strengthened as follows: for some positive $\delta_{0}<\Delta_{0}$ and $\Delta_{1}>\delta_{1}$, and for some sufficiently small $\eta>0$, the level sets are intertwined as follows:

$$
\begin{aligned}
\left\{x: V_{1}(x) \leq \delta_{1}\right\} & \subset\left\{x: V_{0}(x) \leq \delta_{0}\right\} \\
\left\{x: V_{0}(x) \leq \delta_{0}\right\}+B(0, \eta) & \subset\left\{x: V_{1}(x) \leq \Delta_{1}\right\} \\
\left\{x: V_{1}(x)<\Delta_{1}\right\} & \subset\left\{x: V_{0}(x)<\Delta_{0}\right\} .
\end{aligned}
$$

We also assume universal bounds of the form

$$
V_{1}(x) \leq \alpha_{1}+\beta_{1}|x|^{2}, \quad V_{0}(x) \geq \beta_{2}|x|^{2} \forall x \in \mathbb{R}^{n},
$$

for certain positive constants (other types of growth bounds could also serve).

The point in the following is that it is the Lyapunov functions that are blended first, from which is derived a blending of the feedbacks.

Theorem 24. There exists a semiconcave clf $V$ of the form

$$
V(x):=\min \left\{V_{0}(x), c V_{1}(x)+d\right\}
$$

which induces a steepest descent feedback $k$ whose value at every point $x$ is either $k_{0}(x)$ or $k_{1}(x)$, that agrees with $k_{1}$ on the set $\left\{x: V_{1}(x) \geq \Delta_{1}\right\}$ and with $k_{0}$ on the set $\left\{x: V_{0}(x) \leq \delta_{0}\right\}$, and that globally stabilizes the system in the sample-and-hold sense.

Proof. We begin by establishing 
Lemma 1. There exist positive constants $c$ and $d$ such that

$$
\begin{aligned}
V_{0}(x) \leq \delta_{0} & \Longrightarrow c V_{1}(x)+d>\delta_{0} \\
V_{1}(x) \geq \Delta_{1} & \Longrightarrow c V_{1}(x)+d<V_{0}(x) .
\end{aligned}
$$

We prove this by first picking $d>\delta_{0}$ so that $V_{0}(x)>d$ for all $x$ satisfying $V_{1}(x) \geq \Delta_{1}$; this is possible because of (46). The first desired implication evidently holds for any positive $c$, since $V_{1}$ is nonnegative. Note that (by choice of d) the function

$$
x \mapsto \frac{V_{0}(x)-d}{\alpha_{1}+\beta_{1}|x|^{2}}
$$

is strictly positive on the set $\left\{V_{1} \geq \Delta_{1}\right\}$, and, in the limit as $|x| \rightarrow \infty$, is bounded below by $\beta_{2} / \beta_{1}>0$ (as a consequence of (48)). This implies the existence of a constant $c>0$ such that

$$
V_{1}(x) \geq \Delta_{1} \Longrightarrow \frac{V_{0}(x)-d}{\alpha_{1}+\beta_{1}|x|^{2}}>c .
$$

It follows now from (48) that $c$ and $d$ have the properties stated in the Lemma.

We now define

$$
k(x):= \begin{cases}k_{0}(x) & \text { if } V_{0}(x) \leq c V_{1}(x)+d \\ k_{1}(x) & \text { if } V_{0}(x) \geq c V_{1}(x)+d\end{cases}
$$

(the ambiguity when equality holds may be resolved by either choice). We must verify that $k_{0}$ and $k_{1}$ are indeed available (that is, defined) when they are called upon in this definition.

Case 1. When $V_{1}(x) \geq \Delta_{1}$, then $c V_{1}(x)+d<V_{0}(x)$ (by Lemma 1 ), so that $k_{1}(x)$, which is defined for such $x$, is available as required by the definition of $k$.

Case 2. When $V_{0}(x) \leq \delta_{0}$, then $c V_{1}(x)+d>\delta_{0} \geq V_{0}(x)$ (by Lemma 1), so that $k_{0}(x)$, which is defined for such $x$, is available as required by the definition of $k$.

Case 3. For the remaining values of $x$, we have both $\delta_{0}<V_{0}(x)$ and $V_{1}(x)<\Delta_{1}$; it follows from the second inequality, together with $(47)$, that $k_{0}(x)$ is defined; it follows from the first inequality, together with (45), that $k_{1}(x)$ is defined. Since both $k_{0}(x)$ and $k_{1}(x)$ are defined, then $k(x)$ is too.

Note that this analysis shows that $k$ agrees with $k_{1}$ on the set $\left\{V_{1} \geq \Delta_{1}\right\}$, and with $k_{0}$ on the set $\left\{V_{0} \leq \delta_{0}\right\}$. We now show that $k$ is a steepest descent feedback for a certain semiconcave clf $V$, from which it follows that $k$ stabilizes the system (see Theorem 21).

We define

$$
V(x):=\min \left\{V_{0}(x), c V_{1}(x)+d\right\},
$$

a function which is easily seen to be continuous, positive definite, and proper.

Lemma 2. $V$ is semiconcave on $\mathbb{R}^{n} \backslash\{0\}$.

We wish to verify that property SC of Section 5 holds at every point $x \neq 0$. To prove this, it suffices to consider points

$$
x \in\left\{y: V_{1}(y) \leq \delta_{1}\right\},
$$

since $V_{1}$ (and therefore $c V_{1}+k$ ) satisfies SC on the complement of this set, $V_{0}$ satisfies SC on $\mathbb{R}^{n} \backslash\{0\}$, and since the pointwise minimum of two functions both satisfying $\mathrm{SC}$ at a point also satisfies SC at the point (see Theorem 14). Accordingly, let $x \neq 0$ satisfy $V_{1}(x) \leq \delta_{1}$. Then $V_{0}(x) \leq \delta_{0}$ by (45), which yields

$$
c V_{1}(x)+d>\delta_{0} \geq V_{0}(x),
$$

by Lemma 1 . It follows that locally, $V$ coincides with $V_{0}$, whence $V$ satisfies SC at $x$. The Lemma is proved.

The final step in the proof is to show that $k$ satisfies the infinitesimal decrease condition for $V$ at every $x \neq 0$, in the sense of (38): It follows then from Theorem 21 that $k$ stabilizes the system.

When $V_{0}(x)<c V_{1}(x)+d$, we have $V=V_{0}$ locally and $k=k_{0}$. But (by Lemma 1) we also have $V_{1}(x)<\Delta_{1}$, so that $V_{0}(x)<\Delta_{0}$ (by (47)), and infinitesimal decrease for $V_{0}, k_{0}$ holds; that is

$$
\exists \zeta \in \partial_{C} V_{0}(x) \text { such that }\left\langle\zeta, f\left(x, k_{0}(x)\right\rangle<-W_{0}(x),\right.
$$

where $W_{0}$ is the decrease rate function associated to $V_{0}$. Since $V=V_{0}$ locally, we have $\zeta \in \partial_{C} V(x)$, and since $k=k_{0}$, we deduce infinitesimal decrease for $V, k$ at $x$ (with rate $\left.W_{0}(x)\right)$.

When $V_{0}(x)>c V_{1}(x)+d$, we have $V=c V_{1}+d$ locally and $k=k_{1}$. We claim that $V_{1}(x)>\delta_{1}$. Indeed, if we have $V_{1}(x) \leq \delta_{1}$, then $V_{0}(x) \leq \delta_{0}$ (by (45)), whence

$$
c V_{1}(x)+d>\delta_{0} \geq V_{0}(x)
$$

(by Lemma 1), a contradiction that proves the claim. It follows now that $V_{1}, k_{1}$ satisfies infinitesimal decrease at $x$ :

$$
\exists \zeta \in \partial_{C} V_{1}(x) \text { such that }\left\langle\zeta, f\left(x, k_{1}(x)\right)\right\rangle<-W_{1}(x),
$$

where $W_{1}$ is the decrease rate function associated to $V_{1}$. Since

$$
\partial_{C}\left(c V_{1}+d\right)=c \partial_{C} V_{1},
$$

and since $V=c V_{1}+d$ locally, we deduce $c \zeta \in \partial_{C} V(x)$. We have $k(x)=k_{1}(x)$, whence

$$
\langle c \zeta, f(x, k(x))\rangle<-c W_{1}(x),
$$

which shows that $V, k$ satisfies infinitesimal decrease at $x$ with rate $c W_{1}(x)$.

The remaining case is that in which $V_{0}(x)=c V_{1}(x)+d$. In that case, nonsmooth calculus provides the formula

$$
\partial_{C} V(x)=\operatorname{co}\left\{\partial_{C} V_{0}(x), c \partial_{C} V_{1}(x)\right\} .
$$

Suppose first that we have elected to define $k(x)=k_{0}(x)$. Then the required infinitesimal decrease for $V, k$ will follow from that for $V_{0}, k_{0}$ as in the argument above; we know this holds provided $V_{0}(x)<\Delta_{0}$, which we proceed to verify: We have $V_{1}(x)<\Delta_{1}$ in view of Lemma 1 , so that $V_{0}(x)<\Delta_{0}$ by $(47)$.

If, alternatively, we have elected to define $k(x)=k_{1}(x)$, then the required infinitesimal decrease for $V, k$ will follow as above from that for $V_{1}, k_{1}$, which holds provided $V_{1}(x)>\delta_{1}$, which we now verify: We have $V_{0}(x)>\delta_{0}$ in view of Lemma 1 , so that $V_{1}(x)>\delta_{1}$ by $(45)$. 
We remark that the proof provided the rate function $\min \left\{W_{0}, c W_{1}\right\}$ for the clf $V$ and its steepest descent feedback $k$.

\section{SLIDING-MODE CONTROL}

Sliding-mode control is a well-known stabilization technique which has generated a large literature: we refer to the monographs of Utkin (1992) and Edwards and Spurgeon (1998) and overviews of the field by Slotine and Li (1991) and by Young et al. (1999).

Under sliding-mode control, the state is first driven towards a subset $\Sigma$ of the state space, the sliding set, which possesses a strong stability property. Subsequently, the state trajectory remains near $\Sigma$ and moves asymptotically to the origin.

Sliding-mode feedbacks commonly take the form

$$
x \mapsto g(x)+k(x)
$$

in which $g(x)$ is smooth and $k(x)$ is a discontinuous feedback with values in $U$. The purpose of the discontinuous term $k(x)$ is to force the state to approach $\Sigma$ at a uniformly positive rate. The continuous term $g(x)$ can be thought of as a preliminary configuration of the system.

Since, if ever the state trajectory departs from $\Sigma$, the controller drives it back towards $\Sigma$, we expect that the state trajectory attains $\Sigma$ and then remains in it in some sense. If sliding-mode control is implemented digitally, with a high sample rate, the control values generated by the control law are typically observed to switch rapidly, after the state trajectory first crosses $\Sigma$, in such a manner that the state trajectory remains close to $\Sigma$, and lies in $\Sigma$, in the limit as the sample period tends to zero.

The classical approach to analyzing the feedback response of a system under sliding-mode control assumes that the state approaches and attains the sliding set $\Sigma$ in an initial phase, and remains in it thereafter in the final phase. In the final phase, reduced (or 'equivalent') dynamics pertain, and traditional techniques are employed to analyze stability.

On the face of it, this would seem to come perilously close to the thin set fallacy (since, in fact, $\Sigma$ is usually a set of measure zero), which is cause for concern when discontinuous feedbacks are involved (see Section 7). What makes the technique work in this case is that the sliding set is strongly stable, so that simply remaining near $\Sigma$ suffices to drive the state to 0. Clearly, however, any analysis based on the supposition that, in the final phase, the state remains precisely in the sliding set can only have heuristic value, and necessarily precludes treating certain types of error

In Clarke and Vinter (2009), certain Lyapunov functions, together with steepest descent feedbacks implemented in the sample-and-hold sense, are used in the analysis of sliding-mode control. A distinctive feature of this approach is that it takes account, from the outset, of implementation constraints, and gives conditions under which these controllers are stabilizing, in the presence not only of large disturbances, but also of modeling, actuator and observation errors. We obtain in this way the first rigorous error analysis of the procedure.

Theorem 25 below pertains to a model featuring 'delayfree, zero-order hold' digital control implementation, in which the state is measured and the corresponding control value is calculated instantaneously at each sample time, and this control value is applied until the next sampling; that is, sample-and-hold. The focus on a particular implementation scheme might seem restrictive, but this is not the case. Indeed, as shown in Clarke and Vinter (2009), many practical implementation schemes (digital control with time delay, filtering, hysteresis and/or regularization of the discontinuous controller) can be interpreted as a standard sample-and-hold scheme with measurement and/or actuator error, and are therefore covered by our model.

\section{System description}

Given the initial condition $x_{0}$, a partition $\pi=\left\{t_{i}\right\}_{i \geq 0}$ of $[0, \infty)$, and a two-part feedback $g, k$ as in (49), we generate a state trajectory $x(\cdot)$ as follows:

$$
\left.\begin{array}{rl}
x(0) & =x_{0} \\
x^{\prime}(t) & =f\left(x(t), u_{i}, d(t)\right) \text { a.e. } t \in\left[t_{i}, t_{i+1}\right],
\end{array}\right\}
$$

where the constant control value $u_{i}$ applied on the partition interval $\left[t_{i}, t_{i+1}\right]$ is given by

$$
u_{i}=a_{i}+g\left(x\left(t_{i}\right)+m_{i}\right)+k\left(x\left(t_{i}\right)+m_{i}\right) \quad \forall i \geq 0,
$$

and where $d(\cdot):[0, \infty) \rightarrow \mathbb{R}^{k}$ is a measurable function having values in a given subset $D$ of $\mathbb{R}^{k}$. Note that $f$ now depends on the additional 'disturbance variable' $d$.

The sequences $\left\{a_{i}\right\}$ and $\left\{m_{i}\right\}$ describe the $n$-vector actuator errors and $m$-vector measurement errors at successive sample instants, respectively, and $d(\cdot)$ is a disturbance signal. Because the feedback law is applied in a sampleand-hold manner, a physically meaningful state $x(\cdot)$ is generated by the scheme, depending of course on the initial state $x_{0}$ and the partition, the values of the feedback, the errors $m_{i}$ and $a_{i}$, and the disturbance $d(\cdot)$. In contrast to earlier sections, there is no single trajectory generated by the partition and the feedback; stabilization is now understood in the robust sense that all possible trajectories generated this way go to zero (for suitably fine partitions, for sufficiently small errors).

The hypotheses on the data are: continuity and linear growth of $f$ and $g$, compactness of $U$ and $D$, local Lipschitz behavior of $f$ with respect to the state.

\section{Lyapunov functions for sliding-mode control}

We assume that the feedback design has been carried out in ignorance of the measurement and actuator errors, and on the basis of a possibly inaccurate nominal dynamic model:

$$
x^{\prime}=f_{0}(x, u, d)
$$

in which the function $f_{0}$ may differ from the true dynamic function $f$. The only hypothesis imposed on $f_{0}$ is that it be continuous. 
We proceed to introduce not one, but two Lyapunov functions $V_{1}: R^{n} \rightarrow[0, \infty)$ and $V_{2}: R^{n} \rightarrow[0, \infty)$, a decrease rate function $W: R^{n} \rightarrow[0, \infty)$ associated with $V_{2}$, and a subset $\Sigma \subset R^{n}$ of the state space, the sliding set. $\Sigma$ is assumed to be a closed set containing the origin.

$V_{1}$ will be used to capture the property that the slidingmode control drives the state arbitrarily close to $\Sigma$, in finite time. $V_{2}$ is associated with the subsequent motion of the state to a neighbourhood of the origin. $V_{1}, V_{2}$ and $W$ will be required to satisfy the following conditions.

(LF1): $V_{1}$ is a continuous nonnegative function, and $V_{1}(x)=0$ if and only if $x \in \Sigma$. Furthermore, the restriction of $V_{1}$ to $R^{n} \backslash \Sigma$ is continuously differentiable, and there exists $\omega_{1}>0$ such that

$$
\begin{aligned}
\left.\left\langle\nabla V_{1}(x), f_{0}(x, g(x)+k(x), d)\right)\right\rangle & \leq-\omega_{1} \\
& \forall x \in R^{n} \backslash \Sigma, d \in D .
\end{aligned}
$$

Note that $V_{1}$ is not assumed to be differentiable at points in $\Sigma$.

Now we define the set $F_{0}(x)$ as follows:

$$
\left\{\lim _{i \rightarrow \infty} f_{0}\left(x, g(x)+k\left(x_{i}\right), d\right): x_{i} \rightarrow x, d \in D\right\} .
$$

We may think of $F_{0}(x)$ as consisting of all possible velocity values $x^{\prime}$ when the state is at $x$ (in limiting terms, and for the nominal dynamics given by $\left.f_{0}\right)$. Note that $F_{0}(x)$ reduces to

$$
f_{0}(x, g(x)+k(x), D)
$$

if $k$ is continuous at $x$. Another case of special interest is that in which $k(x)$ takes a given value $k_{+}$everywhere on, or to one side of, a given sliding hypersurface $\Sigma$ of dimension $n-1$, and a value $k_{-}$on the opposite side. Then, at any point $x$ of $\Sigma$, we have

$$
\begin{aligned}
F_{0}(x)=\left\{f_{0}\left(x, g(x)+k_{+}, d\right): d \in D\right\} & \\
& \cup\left\{f_{0}\left(x, g(x)+k_{-}, d\right): d \in D\right\} .
\end{aligned}
$$

The set $F_{0}(x)$ is used to express the decrease condition satisfied by $V_{2}$.

(LF2): $V_{2}$ and $W$ are continuous nonnegative functions such that $V_{2}(0)=W(0)=0$ and

$$
V_{2}(x)>0 \text { and } W(x)>0 \text { for } x \in \Sigma \backslash\{0\} .
$$

Furthermore, the restriction of $V_{2}$ to $R^{n} \backslash\{0\}$ is continuously differentiable, and

$$
\max _{w \in F_{0}(x)}\left\langle\nabla V_{2}(x), w\right\rangle<-W(x) \quad \text { for all } x \in \Sigma \backslash\{0\} .
$$

Observe that (LF1) and (LF2) incorporate variants of the usual infinitesimal decrease condition of clf's. That of (LF2) is stated with the help of $F_{0}$ because it would not make sense to simply require, for example, that the inner product

$$
\left\langle\nabla V_{2}(x), f(x, g(x)+k(x), d)\right\rangle
$$

be negative when $x$ lies in $\Sigma$. The reason for this is that the set $\Sigma$ may be thin, and $k$ may be discontinuous; an implementation might never actually evaluate $k$ at any points in $\Sigma$, so that the values of the inner product on merely the sliding set cannot in themselves assure the required stabilization.

We require one more property of the Lyapunov pair:

(LF3): $V_{1}+V_{2}$ is proper; that is, for any $c$, the following level set is bounded:

$$
\left\{x: V_{1}(x)+V_{2}(x) \leq c\right\} .
$$

Sufficient conditions for stabilization

The theorem below is taken from Clarke and Vinter (2009). It asserts that if the actuator and measurement errors are sufficiently small (the proof gives explicit bounds), if the modeling error between $f$ and $f_{0}$ (matched to the gradients of $V_{1}$ and $V_{2}$ ) is sufficiently small, and if the partition size is small enough (or equivalently, the sampling rate high enough), then sample-and-hold stabilization takes place.

We define the set $M(x)$ by

$$
\left\{\lim _{i \rightarrow \infty}\left(f-f_{0}\right)\left(x, g(x)+k\left(x_{i}\right), d\right): x_{i} \rightarrow x, d \in D\right\} .
$$

We may think of $M(x)$ as consisting of the relevant modeling error (in limiting terms) at the state $x$.

Theorem 25. Let $V_{1}, V_{2}, W$, and $\Sigma$ satisfy hypotheses (LF1)-(LF3). Choose any numbers

$$
R>r>0, \omega \in\left(0, \omega_{1}\right), \epsilon>0 .
$$

Then there exist positive numbers

$$
C, e_{m}, e_{a}, \delta, e_{1}, e_{2}, T
$$

(where $C$ does not depend on $r$ ) with the following properties:

Take any sequences $\left\{m_{i}\right\}$ and $\left\{a_{i}\right\}$ in $R^{m}$ and $R^{n}$ respectively, partition $\left\{t_{i}\right\}$, measurable function $d:[0, \infty) \rightarrow D$ and $x_{0} \in B(0, R)$ satisfying

$$
\left|m_{i}\right| \leq e_{m}, \quad\left|a_{i}\right| \leq e_{a},\left|t_{i+1}-t_{i}\right| \leq \delta \quad \text { for all } i .
$$

Suppose in addition that the two following bounds on modeling error hold:

$$
\begin{array}{r}
\left|\left\langle\nabla V_{1}(x), f(x, g(x)+k(x), d)-f_{0}(x, g(x)+k(x), d)\right\rangle\right| \\
\leq e_{1} \quad \forall x \in B(0, C) \backslash \Sigma, d \in D, \quad(51)
\end{array}
$$

and

$$
\begin{aligned}
\left|\left\langle\nabla V_{2}(x), w\right\rangle\right| & \leq e_{2} \\
\forall w & \in M(x), x \in(B(0, C) \cap \Sigma) \backslash\{0\} .
\end{aligned}
$$

Let $x(\cdot)$ be any state trajectory generated by (50) (such trajectories exist). Then

$$
|x(t)| \leq C \quad \forall t \geq 0, x(t) \in B(0, r) \quad \forall t \geq T .
$$

Furthermore,

$$
d_{\Sigma}(x(t)) \leq \epsilon \quad \text { for all } t \in\left[V_{1}(x(0)) / \omega, \infty\right),
$$

where $d_{\Sigma}$ is the Euclidean distance to the set $\Sigma$.

An example

We now illustrate in a simple example how sliding-mode control can yield robust feedback stabilization in the 
presence of arbitrarily large modeling error (at the price of large and active control laws); we also interpret in our context the known issue of 'matching' the errors.

The setting is a familiar one in texts on sliding-mode control (see for example Slotine and Li (1991)). We take $n=2$ and denote points in state space by $(x, y)$. The dynamics are given by

$$
x^{\prime}(t)=y(t), y^{\prime}(t)=h(x(t))+u(t) .
$$

The goal is to stabilize the state to the origin by means of continuous + switching state feedback, where the switching term is bounded in magnitude by some constant $L>0$.

The choice of sliding set is

$$
\Sigma:=\{(x, y): x+y=0\},
$$

a choice motivated by the fact that if the $(x, y)$ could be restricted to a neighborhood of $\Sigma$ (by some discontinuous feedback strategy), the dynamics would then imply $\dot{x} \approx$ $-x$, which in turn seems to imply the stabilization of $x$ to 0 . As for the component $y$ of the state, note that the corresponding differential equation

$$
\dot{y}(t)=h(x(t))+u(t)
$$

leaves the fate of $y$ somewhat in doubt; of course, this differential equation is irrelevant on the sliding set itself, except (possibly) as a limiting idealization. On the other hand, the relation $y(t) \approx-x(t)$ tends to confirm that $y$ should converge to 0 too. Given that in practice the state $(x, y)$ will not be exactly in $\Sigma$, a rigorous analysis requires a different approach; Theorem 25 provides this.

We take a nominal dynamic system having the same structure:

$$
x^{\prime}(t)=y(t), y^{\prime}(t)=h_{0}(x(t))+u(t) .
$$

where the modeling error $h-h_{0}$ may be large. (However, we suppress the disturbance signal, for ease of exposition.) It is assumed that $h$ and $h_{0}$ are continuous and have linear growth, and that $h-h_{0}$ is globally bounded; we also assume that $h$ is Lipschitz on bounded sets.

We wish to place ourselves in the general framework considered by Theorem 25 , for $n=2, m=1$ and

$$
f(x, y, u)=[y, h(x)+u]^{T}, U=[-L, L] .
$$

Thus we seek a feedback

$$
(x, y) \mapsto g(x, y)+k(x, y),
$$

where $k(x, y) \in[-L, L]$. The nominal function $f_{0}$ is the same as $f$, but with $h$ replaced by $h_{0}$.

We first choose Lyapunov functions in accordance with (LF1)-(LF3). A natural choice for $V_{1}$ is

$$
V_{1}(x, y):=|x+y|,
$$

which is continuous, zero precisely on $\Sigma$, and continuously differentiable on $R^{2} \backslash \Sigma$. The decrease condition required in hypothesis (LF1) of the Theorem becomes

$$
\frac{x+y}{|x+y|}\left\{h_{0}(x)+y+g(x, y)+k(x, y)\right\} \leq-\omega_{1} .
$$

This suggests taking

$$
g(x, y)=-h_{0}(x)-y, k(x, y)=-L \operatorname{sgn}(x+y) .
$$

(The value assigned to $\operatorname{sgn}(0)$ will be immaterial.) With these choices, we see that $\omega_{1}$ can be taken to be $L$.

There are many possible choices for $V_{2}$, but a function depending only upon $x$ suggests itself, for the reason that $\nabla V_{2}$ then has a zero inner product with $f-f_{0}$ : the Lyapunov function is 'matched' to the modeling error. This automatically assures that the bound (52) in the statement of the theorem will be satisfied. We take $V_{2}(x, y)=x^{2}$.

With these choices, we see that (LF2) and (LF3) are satisfied, for $W(x, y)=x^{2}$.

Proposition 26. Suppose that $L$ is taken larger than

$$
\left\|h-h_{0}\right\|_{\infty}:=\sup _{x \in R^{n}}\left|\left(h-h_{0}\right)(x)\right| .
$$

Then, for any $0<r<R$, for all sufficiently small levels of actuator and measurement error, and for all sufficiently fine partitions, the feedback given above stabilizes initial points in $B(0, R)$ to $B(0, r)$.

Proof. (Sketch) In order to apply Theorem 25, we set $\omega_{1}=L$ and then take any $\omega$ in the open interval

$$
\left(0, L-\left\|h-h_{0}\right\|_{\infty}\right) .
$$

The left side of (51) is bounded by $\left\|h-h_{0}\right\|_{\infty}$, which can be shown to provide a suitable choice of $e_{1}$ (for all sufficiently small values of $e_{a}, e_{m}$ and $\delta$ ). As mentioned, (52) holds automatically. The Theorem applies and yields the required conclusion.

\section{REFERENCES}

F. Ancona and A. Bressan. Patchy vector fields and asymptotic stabilization. ESAIM:COCV, 4:445-471, 1999.

Z. Artstein. Stabilization with relaxed controls. Nonlinear Analysis TMA, 7:1163-1173, 1983.

A. Astolfi. Discontinuous control of the Brockett integrator. European J. Control, 4:49-63, 1998.

A. Bacciotti and L. Rosier. Lyapunov functions and stability in control theory, volume 267 of Lecture Notes in Control and Information Sciences. Springer-Verlag, London, 2001.

M. Bardi and I. Capuzzo-Dolcetta. Optimal control and viscosity solutions of Hamilton-Jacobi-Bellman equations. Birkhäuser, Boston, 1997.

L. D. Berkovitz. Optimal feedback controls. SIAM J. Control Optim., 27:991-1006, 1989.

R. W. Brockett. Asymptotic stability and feedback stabilization. In R.W. Brockett, R.S. Millman, and H.J. Sussmann, editors, Differential Geometric Control Theory, pages 181-191. Birkhäuser, Boston, 1983.

B. Brogliato. Nonsmooth Mechanics. Springer-Verlag, London, 1999.

P. Cannarsa and C. Sinestrari. Semiconcave Functions, Hamilton-Jacobi Equations, and Optimal Control. Birkhäuser, Boston, 2004.

F. H. Clarke. Maximum principles without differentiability. Bulletin Amer. Math. Soc., 81:219-222, 1975.

F. H. Clarke. The maximum principle under minimal hypotheses. SIAM J. Control Optim., 14:1078-1091, 1976. 
F. H. Clarke. Optimization and Nonsmooth Analysis. Wiley-Interscience, New York, 1983. Republished as vol. 5 of Classics in Applied Mathematics, SIAM, 1990.

F. H. Clarke. Methods of Dynamic and Nonsmooth Optimization. S.I.A.M., Philadelphia, 1989. Regional Conference Series in Applied Mathematics vol. 57.

F. H. Clarke. Necessary Conditions in Dynamic Optimization. Memoirs of the Amer. Math. Soc., 173(816), 2005.

F. H. Clarke and R. J. Stern. State constrained feedback stabilization. SIAM J. Control Optim., 42:422-441, 2003.

F. H. Clarke and R. J. Stern. Lyapunov and feedback characterizations of state constrained controllability and stabilization. Systems and Control Letters, 54:747-752, 2005.

F. H. Clarke and R. B. Vinter. Applications of optimal multiprocesses. SIAM J. Control Optim., 27:1048-1071, 1989

F. H. Clarke and R.B. Vinter. Stability analysis of slidingmode feedback control. J. Cybernetics and Control, 38: 1169-1192, 2009

F. H. Clarke, Yu. S. Ledyaev, and A. I. Subbotin. Universal feedback control via proximal aiming in problems of control under disturbances and differential games. Rapport CRM (Univ. de Montréal), 2386, 1994.

F. H. Clarke, Yu. S. Ledyaev, E. D. Sontag, and A. I Subbotin. Asymptotic controllability implies feedback stabilization. IEEE Trans. Aut. Control, 42:1394-1407, 1997.

F. H. Clarke, Yu. S. Ledyaev, R. J. Stern, and P. R Wolenski. Nonsmooth Analysis and Control Theory. Graduate Texts in Mathematics, vol. 178. SpringerVerlag, New York, 1998.

F. H. Clarke, Yu. S. Ledyaev, L. Rifford, and R. J. Stern. Feedback stabilization and Lyapunov functions. SIAM J. Control Optim., 39:25-48, 2000.

F. H. Clarke, L. Rifford, and R. J. Stern. Feedback in state constrained optimal control. ESAIM Control Optim. Calc. Var., 7:97-133, 2002.

J.-M. Coron. Mathematical surveys and monographs. In Control and Nonlinearity, volume 136. American Mathematical Society, Providence, 2007.

J.-M. Coron and L. Rosier. A relation between continuous time-varying and discontinuous feedback stabilization. J. Math. Syst., Estimation, Control, 4:67-84, 1994.

J.-M. Coron, L. Praly, and A. Teel. Feedback stabilization of nonlinear systems: Sufficient conditions and Lyapunov and input-output techniques. In A. Isidori, editor, Trends in Control. Springer-Verlag, New York, 1995.

C. Edwards and S. K. Spurgeon. Sliding Mode Control. Taylor and Francis, London, 1998.

A. F. Filippov. Differential Equations with Discontinuous Righthand Sides. Kluwer Academic Publishers, Dordrecht, 1988.

F. A. C. C. Fontes and L. Magni. Min-max model predictive control of nonlinear systems using discontinuous feedbacks. New directions on nonlinear control. IEEE Trans. Automt. Control, 48:1750-1755, 2003.

R. A. Freeman and P. V. Kokotović. Robust nonlinear control design. State space and Lyapunov techniques. Systems and Control: Foundations and Applications. Birkhäuser, Boston, 1996.
A. Isidori. Nonlinear Control Systems. Springer-Verlag, 1995. 3rd Ed.

C. M. Kellett and A. R. Teel. On the robustness of $\mathcal{K} \mathcal{L}$ stability for difference inclusions: smooth discrete-time Lyapunov functions. SIAM J. Control Optim., 44:777800, 2005.

A. B. Khurzhanski, I. M. Mitchell, and P. Varaiya. Optimization techniques for state-constrained control and obstacle problems. J. Optim. Theory Appl., 128:499$521,2006$.

N. N. Krasovskii and A. I. Subbotin. Game-Theoretical Control Problems. Springer-Verlag, New York, 1988.

Yu. S. Ledyaev and E. D. Sontag. A Lyapunov characterization of robust stabilization. Nonlinear Analysis, 37: 813-840, 1999.

Yu. S. Ledyaev and R. B. Vinter. A Lyapunov characterization of robust stabilization. Proc. Steklov Inst. Math., 268:222-241, 2010.

S. Nobakhtian and R. J. Stern. Universal near-optimal feedbacks. J. Optim. Theory Appl., 107:89-122, 2000.

Yu. V. Orlov. Discontinuous Systems. Springer, London, 2009 .

L. S. Pontryagin, V. G. Boltyanskii, R. V. Gamkrelidze, and E. F. Mischenko. The Mathematical Theory of Optimal Processes. Wiley-Interscience, New York, 1962.

C. Prieur and E. Trélat. Robust optimal stabilization of the Brockett integrator via a hybrid feedback. Math. Control Signals Systems, 17:201-216, 2005.

L. Rifford. Existence of Lipschitz and semiconcave controlLyapunov functions. SIAM J. Control Optim, 39:10431064, 2000.

L. Rifford. Singularities of viscosity solutions and the stabilization problem in the plane. Indiana Univ. Math. J., 52:1373-1396, 2003.

L. Rifford. Stratified semiconcave control-Lyapunov functions and the stabilization problem. Ann. Inst. $H$. Poincaré Anal. Non Linéaire, 22:343-384, 2005.

L. Rifford. On the existence of local smooth repulsive stabilizing feedbacks in dimension three. J. Differential Equations, 226:429-500, 2006.

E. P. Ryan. On Brockett's condition for smooth stabilizability and its necessity in a context of nonsmooth feedback. SIAM J. Control Optim., 32:1597-1604, 1994.

J-J. E. Slotine and Weiping Li. Applied Nonlinear Control. Prentice-Hall, New Jersey, 1991.

E. D. Sontag. A Lyapunov-like characterization of asymptotic controllability. SIAM J. Control Optim., 21:462471,1983

E. D. Sontag. Mathematical Control Theory. Texts in Applied Mathematics, vol.6. Springer-Verlag, New York, 1990. (Second Edition, 1998).

E. D. Sontag. Stability and stabilization: discontinuities and the effect of disturbances. In F. H. Clarke and R. J. Stern, editors, Nonlinear Analysis, Differential Equations and Control (NATO ASI, Montreal 1998), pages 551-598. Kluwer Acad. Publ., Dordrecht, 1999.

V. I. Utkin. Sliding Modes in Control and Optimization. Springer, Berlin, 1992.

R. B. Vinter. Optimal Control. Birkhäuser, Boston, 2000.

K.D. Young, V.I. Utkin, and U. Özgüner. A control engineer's guide to sliding mode control. IEEE Trans. Control Sys. Tech., 7:328-342, 1999. 